\title{
Prym Varieties and Teichmüller Curves
}

\section{Citation}

McMullen, Curtis T. 2007. Prym varieties and Teichmüller curves. Duke Mathematical Journal 133(3): 569-590.

\section{Published Version}

http://dx.doi.org/10.1215/S0012-7094-06-13335-5

\section{Permanent link}

http://nrs.harvard.edu/urn-3:HUL.InstRepos:3426331

\section{Terms of Use}

This article was downloaded from Harvard University's DASH repository, and is made available under the terms and conditions applicable to Other Posted Material, as set forth at http:// nrs.harvard.edu/urn-3:HUL.InstRepos:dash.current.terms-of-use\#LAA

\section{Share Your Story}

The Harvard community has made this article openly available.

Please share how this access benefits you. Submit a story.

Accessibility 


\title{
Foliations of Hilbert modular surfaces
}

\author{
Curtis T. McMullen*
}

21 February, 2005

\begin{abstract}
The Hilbert modular surface $X_{D}$ is the moduli space of Abelian varieties $A$ with real multiplication by a quadratic order of discriminant $D>1$. The locus where $A$ is a product of elliptic curves determines a finite union of algebraic curves $X_{D}(1) \subset X_{D}$.

In this paper we show the lamination $X_{D}(1)$ extends to an essentially unique foliation $\mathcal{F}_{D}$ of $X_{D}$ by complex geodesics. The geometry of $\mathcal{F}_{D}$ is related to Teichmüller theory, holomorphic motions, polygonal billiards and Lattès rational maps. We show every leaf of $\mathcal{F}_{D}$ is either closed or dense, and compute its holonomy. We also introduce refinements $T_{N}(\nu)$ of the classical modular curves on $X_{D}$, leading to an explicit description of $X_{D}(1)$.
\end{abstract}

\section{Contents}

$1 \quad$ Introduction . . . . . . . . . . . . . . . . . 1

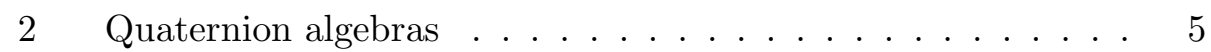

3 Modular curves and surfaces . . . . . . . . . . . . . . . 12

4 Laminations . . . . . . . . . . . . . . . . . . . . 17

5 Foliations of Teichmüller space . . . . . . . . . . . 20

6 Genus two . . . . . . . . . . . . . . 23

7 Holomorphic motions . . . . . . . . . . . . . . 25

8 Quasiconformal dynamics . . . . . . . . . . . . 27

9 Further results . . . . . . . . . . . . . . 30

${ }^{*}$ Research supported in part by the NSF and the Guggenheim Foundation. 


\section{Introduction}

Let $D>1$ be an integer congruent to 0 or $1 \bmod 4$, and let $\mathcal{O}_{D}$ be the real quadratic order of discriminant $D$. The Hilbert modular surface

$$
X_{D}=(\mathbb{H} \times \mathbb{H}) / \operatorname{SL}\left(\mathcal{O}_{D} \oplus \mathcal{O}_{D}^{\vee}\right)
$$

is the moduli space for principally polarized Abelian varieties

$$
A_{\tau}=\mathbb{C}^{2} /\left(\mathcal{O}_{D} \oplus \mathcal{O}_{D}^{\vee} \tau\right)
$$

with real multiplication by $\mathcal{O}_{D}$.

Let $X_{D}(1) \subset X_{D}$ denote the locus where $A_{\tau}$ is isomorphic to a polarized product of elliptic curves $E_{1} \times E_{2}$. The set $X_{D}(1)$ is a finite union of disjoint, irreducible algebraic curves $(\S 4)$, forming a lamination of $X_{D}$. Note that $X_{D}(1)$ is preserved by the twofold symmetry $\iota\left(\tau_{1}, \tau_{2}\right)=\left(\tau_{2}, \tau_{1}\right)$ of $X_{D}$.

In this paper we will show:

Theorem 1.1 Up to the action of $\iota$, the lamination $X_{D}(1)$ extends to a unique foliation $\mathcal{F}_{D}$ of $X_{D}$ by complex geodesics.

(Here a Riemann surface in $X_{D}$ is a complex geodesic if it is isometrically immersed for the Kobayashi metric.)

Holomorphic graphs. The preimage $\widetilde{X}_{D}(1)$ of $X_{D}(1)$ in the universal cover of $X_{D}$ gives a lamination of $\mathbb{H} \times \mathbb{H}$ by the graphs of countably many Möbius transformations. To foliate $X_{D}$ itself, in $\S 6$ we will show:

Theorem 1.2 For any $\left(\tau_{1}, \tau_{2}\right) \notin \widetilde{X}_{D}(1)$, there is a unique holomorphic function

$$
f: \mathbb{H} \rightarrow \mathbb{H}
$$

such that $f\left(\tau_{1}\right)=\tau_{2}$ and the graph of $f$ is disjoint from $\tilde{X}_{D}(1)$.

The graphs of such functions descend to $X_{D}$, and form the leaves of the foliation $\mathcal{F}_{D}(\S 7)$. The case $D=4$ is illustrated in Figure 1 .

Modular curves. To describe the lamination $X_{D}(1)$ explicitly, recall that the Hilbert modular surface $X_{D}$ is populated by infinitely many modular curves $F_{N}$ [Hir], [vG]. The endomorphism ring of a generic Abelian variety in $F_{N}$ is a quaternionic order $R$ of discriminant $N^{2}$.

In general $F_{N}$ can be reducible, and $R$ is not determined up to isomorphism by $N$. In $\S 3$ we introduce a refinement $F_{N}(\nu)$ of the traditional modular curves, such that the isomorphism class of $R$ is constant along 


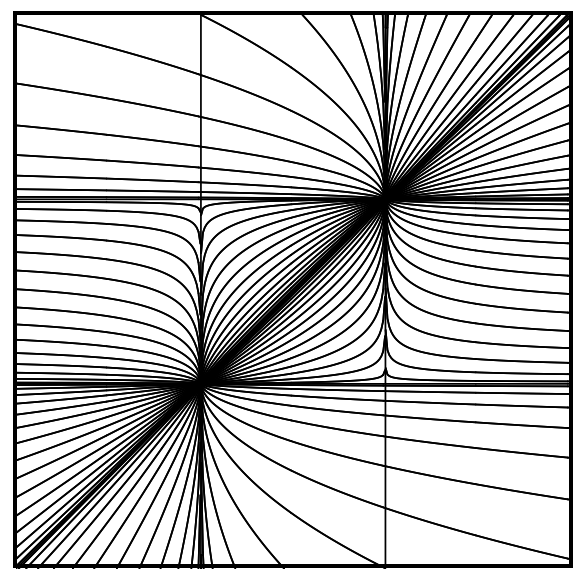

Figure 1. Foliation of the Hilbert modular surface $X_{D}, D=4$.

$F_{N}(\nu)$ and $F_{N}=\bigcup F_{N}(\nu)$. The additional finite invariant $\nu$ ranges in the ring $\mathcal{O}_{D} /(\sqrt{D})$ and its norm satisfies $\mathrm{N}(\nu)=-N \bmod D$. The curves $T_{N}=\bigcup F_{N / \ell^{2}}$ can be refined similarly, and we obtain:

Theorem 1.3 The locus $X_{D}(1) \subset X_{D}$ is given by

$$
X_{D}(1)=\bigcup T_{N}((e+\sqrt{D}) / 2),
$$

where the union is over all integral solutions to $e^{2}+4 N=D, N>0$.

Remark. Although $X_{D}(1)=\bigcup T_{\left(D-e^{2}\right) / 4}$ when $D$ is prime, in general (e.g. for $D=12,16,20,21, \ldots)$ the locus $X_{D}(1)$ cannot be expressed as a union of the traditional modular curves $T_{N}(\S 3)$.

Here is a corresponding description of the lamination $\tilde{X}_{D}(1)$. Given $N>0$ such that $D=e^{2}+4 N$, let

$\Lambda_{D}^{N}=\left\{U=\left(\begin{array}{cc}\mu & b D \\ -a & -\mu^{\prime}\end{array}\right): \begin{array}{l}a, b \in \mathbb{Z}, \mu \in \mathcal{O}_{D}, \operatorname{det}(U)=N \\ \text { and } \mu \equiv \pm(e+\sqrt{D}) / 2 \operatorname{in} \mathcal{O}_{D} /(\sqrt{D})\end{array}\right\}$.

Let $\Lambda_{D}$ be the union of all such $\Lambda_{D}^{N}$. Choosing a real place $\iota_{1}: \mathcal{O}_{D} \rightarrow$ $\mathbb{R}$, we can regard $\Lambda_{D}$ as a set of matrices in $\mathrm{GL}_{2}^{+}(\mathbb{R})$, acting by Möbius transformations on $\mathbb{H}$.

Theorem 1.4 The lamination $\widetilde{X}_{D}(1)$ of $\mathbb{H} \times \mathbb{H}$ is the union of the loci $\tau_{2}=U\left(\tau_{1}\right)$ over all $U \in \Lambda_{D}$. 
We also obtain a description of the locus $X_{D}(E) \subset X_{D}$ where $A_{\tau}$ admits an action of both $\mathcal{O}_{D}$ and $\mathcal{O}_{E}(\S 3)$.

Quasiconformal dynamics. Although its leaves are Riemann surfaces, $\mathcal{F}_{D}$ is not a holomorphic foliation. Its transverse dynamics is given instead by quasiconformal maps, which can be described as follows.

Let $q=q(z) d z^{2}$ be a meromorphic quadratic differential on $\mathbb{H}$. We say a homeomorphism $f: \mathbb{H} \rightarrow \mathbb{H}$ is a Teichmüller mapping relative to $q$ if it satisfies $\bar{\partial} f / \partial f=\alpha q /|q|$ for some complex number $|\alpha|<1$; equivalently, if $f$ has the form of an orientation-preserving real-linear mapping

$$
f(x+i y)=\left(\begin{array}{ll}
a & b \\
c & d
\end{array}\right)\left(\begin{array}{l}
x \\
y
\end{array}\right)=D_{q}(f)\left(\begin{array}{l}
x \\
y
\end{array}\right)
$$

in local charts where $q=d z^{2}=(d x+i d y)^{2}$.

Fix a transversal $\mathbb{H}_{s}=\{s\} \times \mathbb{H}$ to $\widetilde{\mathcal{F}}_{D}$. Any $g \in \mathrm{SL}\left(\mathcal{O}_{D} \oplus \mathcal{O}_{D}^{\vee}\right)$ acts on $\mathbb{H} \times \mathbb{H}$, permuting the leaves of $\widetilde{\mathcal{F}}_{D}$. The permutation of leaves is recorded by the holonomy map

$$
\phi_{g}: \mathbb{H}_{s} \rightarrow \mathbb{H}_{s},
$$

characterized by the property that $g(s, z)$ and $\left(s, \phi_{g}(z)\right)$ lie on the same leaf of $\widetilde{\mathcal{F}}_{D}$.

In $\S 8$ we will show:

Theorem 1.5 The holonomy acts by Teichmüller mappings relative to a fixed meromorphic quadratic differential $q$ on $\mathbb{H}_{s}$. For $s=i$ and $g=\left(\begin{array}{ll}a & b \\ c & d\end{array}\right)$, we have

$$
D_{q}\left(\phi_{g}\right)=\left(\begin{array}{ll}
a & b \\
c & d
\end{array}\right) \in \mathrm{PSL}_{2}(\mathbb{R}) .
$$

On the other hand, for $z \in \partial \mathbb{H}_{s}$ we have

$$
\phi_{g}(z)=\left(a^{\prime} z-b^{\prime}\right) /\left(-c^{\prime} z+d^{\prime}\right) ;
$$

in particular, the holonomy acts by Möbius transformations on $\partial \mathbb{H}_{s}$.

Here $(x+y \sqrt{D})^{\prime}=(x-y \sqrt{D})$. Note that both Galois conjugate actions of $g$ on $\mathbb{R}^{2}$ appear, as different aspects of the holonomy map $\phi_{g}$.

Quantum Teichmüller curves. For comparison, consider an isometrically immersed Teichmüller curve

$$
f: V \rightarrow \mathcal{M}_{g}
$$


generated by a holomorphic quadratic differential $(Y, q)$ of genus $g$. For simplicity assume $\operatorname{Aut}(Y)$ is trivial. Then the pullback of the universal curve $X=f^{*}\left(\mathcal{M}_{g, 1}\right)$ gives an algebraic surface

$$
p: X \rightarrow V
$$

with $p^{-1}(v)=Y$ for a suitable basepoint $v \in V$. The surface $X$ carries a canonical foliation $\mathcal{F}$, transverse to the fibers of $p$, whose leaves map to Teichmüller geodesics in $\mathcal{M}_{g, 1}$. The holonomy of $\mathcal{F}$ determines a map

$$
\pi_{1}(V, v) \rightarrow \operatorname{Aff}^{+}(Y, q)
$$

giving an action of the fundamental group by Teichmüller mappings; and its linear part yields the isomorphism

$$
\pi_{1}(V, v) \cong \operatorname{PSL}(Y, q) \subset \operatorname{PSL}_{2}(\mathbb{R}),
$$

where $\operatorname{PSL}(Y, q)$ is the stabilizer of $(Y, q)$ in the bundle of quadratic differentials $Q \mathcal{M}_{g} \rightarrow \mathcal{M}_{g}$. (See e.g. [V1], [Mc4, §2].)

The foliated Hilbert modular surface $\left(X_{D}, \mathcal{F}_{D}\right)$ presents a similar structure, with the fibration $p: X \rightarrow V$ replaced by the holomorphic foliation $\mathcal{A}_{D}$ coming from the level sets of $\tau_{1}$ on $\widetilde{X}_{D}=\mathbb{H} \times \mathbb{H}$. This suggests that one should regard $\left(X_{D}, \mathcal{A}_{D}, \mathcal{F}_{D}\right)$ as a quantum Teichmüller curve, in the same sense that a 3 -manifold with a measured foliation can be regarded as a quantum Teichmüller geodesic [Mc3].

Question. Does every fibered surface $p: X \rightarrow C$ admit a foliation $\mathcal{F}$ by Riemann surfaces transverse to the fibers of $p$ ?

Complements. We conclude in $\S 9$ by presenting the following related results.

1. Every leaf of $\mathcal{F}_{D}$ is either closed or dense.

2. When $D \neq d^{2}$, there are infinitely many eigenforms for real multiplication by $\mathcal{O}_{D}$ that are isoperiodic but not isomorphic.

3. The Möbius transformations $\Lambda_{D}$ give a maximal top-speed holomorphic motion of a discrete subset of $\mathbb{H}$.

4. The foliation $\mathcal{F}_{4}$ also arises as the motion of the Julia set in a Lattès family of iterated rational maps. 
The link with complex dynamics was used to produce Figure 1.

Notes and references. The foliation $\mathcal{F}_{D}$ is constructed using the connection between polygonal billiards and Hilbert modular surfaces presented in [Mc4]. For more on the interplay of dynamics, holomorphic motions and quasiconformal mappings, see e.g. [MSS], [BR], [Sl], [Mc2], [Sul], [McS], $[\mathrm{EKK}]$ and $[\mathrm{Dou}]$. A survey of the theory of holomorphic foliations of surfaces appears in [Br1]; see also [Br2] for the Hilbert modular case.

I would like to thank G. van der Geer, B. Gross and the referees for useful comments and suggestions.

\section{Quaternion algebras}

In this section we consider a real quadratic order $\mathcal{O}_{D}$ acting on a symplectic lattice $L$, and classify the quaternionic orders $R \subset \operatorname{End}(L)$ extending $\mathcal{O}_{D}$.

Quadratic orders. Given an integer $D>0, D \equiv 0$ or $1 \bmod 4$, the real quadratic order of discriminant $D$ is given by

$$
\mathcal{O}_{D}=\mathbb{Z}[T] /\left(T^{2}+b T+c\right), \quad \text { where } D=b^{2}-4 c .
$$

Let $K_{D}=\mathcal{O}_{D} \otimes \mathbb{Q}$. Provided $D$ is not a square, $K_{D}$ is a real quadratic field. Fixing an embedding $\iota_{1}: K_{D} \rightarrow \mathbb{R}$, we obtain a unique basis

$$
K_{D}=\mathbb{Q} \cdot 1 \oplus \mathbb{Q} \cdot \sqrt{D}
$$

such that $\iota_{1}(\sqrt{D})>0$. The conjugate real embedding $\iota_{2}: K_{D} \rightarrow \mathbb{R}$ is given by $\iota_{2}(x)=\iota_{1}\left(x^{\prime}\right)$, where $(a+b \sqrt{D})^{\prime}=(a-b \sqrt{D})$.

Square discriminants. The case $D=d^{2}$ can be treated similarly, so long as we regard $x=\sqrt{d^{2}}$ as an element of $K_{D}$ satisfying $x^{2}=d^{2}$ but $x \notin \mathbb{Q}$. In this case the algebra $K_{D} \cong \mathbb{Q} \oplus \mathbb{Q}$ is not a field, so we must take care to distinguish between elements of the algebra such as

$$
x=d-\sqrt{d^{2}} \in K_{D},
$$

and the corresponding real numbers

$$
\iota_{1}(x)=d-d=0, \quad \text { and } \quad \iota_{2}(x)=d+d=2 d .
$$

Trace, norm and different. For simplicity of notation, we fix $D$ and denote $\mathcal{O}_{D}$ and $K_{D}$ by $K$ and $\mathcal{O}$. 
The trace and norm on $K$ are the rational numbers $\operatorname{Tr}(x)=x+x^{\prime}$ and $\mathrm{N}(x)=x x^{\prime}$. The inverse different is the fractional ideal

$$
\mathcal{O}^{\vee}=\{x \in K: \operatorname{Tr}(x y) \in \mathbb{Z} \forall y \in \mathcal{O}\} .
$$

It is easy to see that $\mathcal{O}^{\vee}=D^{-1 / 2} \mathcal{O}$, and thus the different $\mathcal{D}=\left(\mathcal{O}^{\vee}\right)^{-1} \subset \mathcal{O}$ is the principal ideal $(\sqrt{D})$. The trace and norm descend to give maps

$$
\operatorname{Tr}, \mathrm{N}: \mathcal{O} / \mathcal{D} \rightarrow \mathbb{Z} / D
$$

satisfying

$$
\operatorname{Tr}(x)^{2}=4 \mathrm{~N}(x) \bmod D .
$$

When $D$ is odd, $\operatorname{Tr}: \mathcal{O} / \mathcal{D} \rightarrow \mathbb{Z} / D$ is an isomorphism, and thus (2.1) determines the norm on $\mathcal{O} / \mathcal{D}$. On the other hand, when $D=4 E$ is even, we have an isomorphism

$$
\mathcal{O} / \mathcal{D} \cong \mathbb{Z} / 2 E \oplus \mathbb{Z} / 2
$$

given by $a+b \sqrt{E} \mapsto(a, b)$, and the trace and norm on $\mathcal{O} / \mathcal{D}$ are given by

$$
\operatorname{Tr}(a, b)=2 a \bmod D, \quad \mathrm{~N}(a, b)=a^{2}-E b^{2} \bmod D .
$$

Symplectic lattices. Now let $L \cong\left(\mathbb{Z}^{2 g},\left(\begin{array}{cc}0 & I \\ -I & 0\end{array}\right)\right)$ be a unimodular symplectic lattice of genus $g$. (This lattice is isomorphic to the first homology group $H_{1}\left(\Sigma_{g}, \mathbb{Z}\right)$ of an oriented surface of genus $g$ with the symplectic form given by the intersection pairing.)

Let $\operatorname{End}(L) \cong \mathrm{M}_{2 g}(\mathbb{Z})$ denote the endomorphism ring of $L$ as a $\mathbb{Z}$-module. The Rosati involution $T \mapsto T^{*}$ on $\operatorname{End}(L)$ is defined by the condition $\langle T x, y\rangle=\left\langle x, T^{*} y\right\rangle$; it satisfies $(S T)^{*}=T^{*} S^{*}$, and we say $T$ is self-adjoint if $T=T^{*}$.

Specializing to the case $g=2$, let $L$ denote the lattice

$$
L=\mathcal{O} \oplus \mathcal{O}^{\vee}
$$

with the unimodular symplectic form

$$
\langle x, y\rangle=\operatorname{Tr}(x \wedge y)=\operatorname{Tr}_{\mathbb{Q}}^{K}\left(x_{1} y_{2}-x_{2} y_{1}\right) .
$$

A standard symplectic basis for $L$ (satisfying $\left\langle a_{i} \cdot b_{j}\right\rangle=\delta_{i j}$ ) is given by

$$
\left(a_{1}, a_{2}, b_{1}, b_{2}\right)=\left((1,0),(\gamma, 0),\left(0,-\gamma^{\prime} / \sqrt{D}\right),(0,1 / \sqrt{D})\right),
$$


where $\gamma=(D+\sqrt{D}) / 2$.

The lattice $L$ comes equipped with a proper, self-adjoint action of $\mathcal{O}$, given by

$$
k \cdot\left(x_{1}, x_{2}\right)=\left(k x_{1}, k x_{2}\right) .
$$

Conversely, any proper, self-adjoint action of $\mathcal{O}$ on a symplectic lattice of genus two is isomorphic to this model (see e.g. [Ru], [Mc7, Thm 4.1]). (Here an action of $R$ on $L$ is proper if it is indivisible: if whenever $T \in \operatorname{End}(L)$ and $m T \in R$ for some integer $m \neq 0$, then $T \in R$.)

Matrices. The natural embedding of $L=\mathcal{O} \oplus \mathcal{O}^{\vee}$ into $K \oplus K$ determines an embedding of matrices

$$
\mathrm{M}_{2}(K) \rightarrow \operatorname{End}(L \otimes \mathbb{Q}),
$$

and hence a diagonal inclusion

$$
K \rightarrow \operatorname{End}(L \otimes \mathbb{Q})
$$

extending the natural action $(2.3)$ of $\mathcal{O}$ on $L$. Every $T \in \operatorname{End}(L \otimes \mathbb{Q})$ can be uniquely expressed in the form

$$
T(x)=A x+B x^{\prime}, \quad A, B \in \mathrm{M}_{2}(K),
$$

where $\left(x_{1}, x_{2}\right)^{\prime}=\left(x_{1}^{\prime}, x_{2}^{\prime}\right)$; and we have

$$
T^{*}(x)=A^{\dagger} x+\left(B^{\dagger}\right)^{\prime} x^{\prime},
$$

where $\left(\begin{array}{ll}a & b \\ c & d\end{array}\right)^{\dagger}=\left(\begin{array}{cc}d & -b \\ -c & a\end{array}\right)$.

The automorphisms of $L$ as a symplectic $\mathcal{O}$-module are given, as a subgroup of $M_{2}(K)$, by

$$
\operatorname{SL}\left(\mathcal{O} \oplus \mathcal{O}^{\vee}\right)=\left\{\left(\begin{array}{ll}
a & b \\
c & d
\end{array}\right) \in\left(\begin{array}{cc}
\mathcal{O} & \mathcal{D} \\
\mathcal{O}^{\vee} & \mathcal{O}
\end{array}\right): a d-b c=1\right\}
$$

Compare [vG, p.12].

Integrality. An endomorphism $T \in \operatorname{End}(L \otimes \mathbb{Q})$ is integral if it satisfies $T(L) \subset L$.

Lemma 2.1 The endomorphism $\phi(x)=a x+b x^{\prime}$ of $K$ satisfies $\phi(\mathcal{O}) \subset \mathcal{O}$ iff $a, b \in \mathcal{O}^{\vee}$ and $a+b \in \mathcal{O}$.

Proof. Since $x-x^{\prime} \in \sqrt{D} \mathbb{Z}$ for all $x \in \mathcal{O}$, the conditions on $a, b$ imply $\phi(x)=a\left(x-x^{\prime}\right)+(a+b) x^{\prime} \in \mathcal{O}$ for all $x \in \mathcal{O}$. Conversely, if $\phi$ is integral, then $\phi(1)=a+b \in \mathcal{O}$, and thus $a\left(x-x^{\prime}\right) \in \mathcal{O}$ for all $x \in \mathcal{O}$, which implies $a \in D^{-1 / 2} \mathcal{O}=\mathcal{O}^{\vee}$. 
Corollary 2.2 The endomorphism $T(x)=k x+\left(\begin{array}{cc}a & b D \\ c & d\end{array}\right) x^{\prime}$ is integral iff we have

$$
a, b, c, d, k \in \mathcal{O}^{\vee} \quad \text { and } \quad k+a, k-d \in \mathcal{O} .
$$

Proof. This follows from the preceding Lemma, using the fact that $k x+d x^{\prime}$ maps $\mathcal{O}^{\vee}$ to $\mathcal{O}^{\vee}$ iff $k x-d x^{\prime}$ maps $\mathcal{O}$ to $\mathcal{O}$.

Quaternion algebras. A rational quaternion algebra is a central simple algebra of dimension 4 over $\mathbb{Q}$. Every such algebra has the form

$$
Q \cong \mathbb{Q}[i, j] /\left(i^{2}=a, j^{2}=b, i j=-j i\right)=\left(\frac{a, b}{\mathbb{Q}}\right)
$$

for suitable $a, b \in \mathbb{Q}^{*}$. Any $q \in Q$ satisfies a quadratic equation

$$
q^{2}-\operatorname{Tr}(q) q+\mathrm{N}(q)=0
$$

where $\operatorname{Tr}, \mathrm{N}: Q \rightarrow \mathbb{Q}$ are the reduced trace and norm.

An order $R \subset Q$ is a subring such that, as an additive group, we have $R \cong \mathbb{Z}^{4}$ and $\mathbb{Q} \cdot R=Q$. Its discriminant is the square integer

$$
N^{2}=\left|\operatorname{det}\left(\operatorname{Tr}\left(q_{i} q_{j}\right)\right)\right|>0,
$$

where $\left(q_{i}\right)_{1}^{4}$ is an integral basis for $R$. The discriminants of a pair of orders $R_{1} \subset R_{2}$ are related by $N_{1} / N_{2}=\left|R_{2} / R_{1}\right|^{2}$.

Generators. We say $V \in \operatorname{End}(L)$ is a quaternionic generator if:

1. $V^{*}=-V$,

2. $V^{2}=-N \in \mathbb{Z}, N \neq 0$,

3. $V k=k^{\prime} V$ for all $k \in K$, and

4. $k+D^{-1 / 2} V \in \operatorname{End}(L)$ for some $k \in K$.

These conditions imply that $Q=K \oplus K V$ is a quaternion algebra isomorphic to $\left(\frac{D,-N}{\mathbb{Q}}\right)$. Conversely, we have:

Theorem 2.3 Any Rosati-invariant quaternion algebra $Q$ with

$$
K \subset Q \subset \operatorname{End}(L \otimes \mathbb{Q})
$$

contains a unique pair of primitive quaternionic generators $\pm V$. 
(A generator is primitive unless $(1 / m) V, m>1$ is also a generator.)

Proof. By a standard application of the Skolem-Noether theorem, we can write $Q=K \oplus K W$ with $0 \neq W^{2} \in \mathbb{Q}$ and $W k=k^{\prime} W$ for all $k \in K$. Then $K W$ coincides with the subalgebra of $Q$ anticommuting with the selfadjoint element $\sqrt{D}$, so it is Rosati-invariant. The eigenspaces of $* \mid K W$ are exchanged by multiplication by $\sqrt{D}$, so up to a rational multiple there is a unique nonzero $V \in K W$ with $V^{*}=-V$. A suitable integral multiple of $V$ is then a generator, and a rational multiple is primitive.

Corollary 2.4 Quaternionic extensions $K \subset Q \subset \operatorname{End}(L)$ correspond bijectively to pairs of primitive generators $\pm V \in \operatorname{End}(L)$.

Generator matrices. We say $U \in M_{2}(K)$ is a quaternionic generator matrix if it has the form

$$
U=\left(\begin{array}{cc}
\mu & b D \\
-a & -\mu^{\prime}
\end{array}\right)
$$

with $a, b \in \mathbb{Z}, \mu \in \mathcal{O}$ and $N=\operatorname{det}(U) \neq 0$.

Theorem 2.5 The endomorphism $V(x)=U x^{\prime}$ is a quaternionic generator iff $U$ is a quaternionic generator matrix.

Proof. By (2.4) the condition $V=-V^{*}$ is equivalent to $U^{\dagger}=-U^{\prime}$, and thus $U$ can be written in the form (2.5) with $a, b \in \mathbb{Q}$ and $\mu \in K$. Assuming $U^{\dagger}=-U^{\prime}$, we have

$$
N=\operatorname{det}(U)=U U^{\dagger}=-U U^{\prime}=-V^{2},
$$

so $V^{2} \neq 0 \Longleftrightarrow \operatorname{det}(U) \neq 0$. The condition that $D^{-1 / 2}(k+V)$ is integral for some $k$ implies, by Corollary 2.2, that the coefficients of $U$ satisfy $a, b \in \mathbb{Z}$ and $\mu \in \mathcal{O}$; and given such coefficients for $U$, the endomorphism $D^{-1 / 2}(k+$ $V)$ is integral when $k=-\mu$.

The invariant $\boldsymbol{\nu}(\boldsymbol{U})$. Given generator matrix $U=\left(\begin{array}{cc}\mu & b D \\ -a & -\mu^{\prime}\end{array}\right)$, let $\nu(U)$ denote the image of $\mu$ in the finite ring $\mathcal{O} / \mathcal{D}$. It is easy to check that

$$
\nu(U)= \pm \nu\left(g^{\prime} U g^{-1}\right)
$$

for all $g \in \operatorname{SL}\left(\mathcal{O} \oplus \mathcal{O}^{\vee}\right)$, and that its norm satisfies

$$
\mathrm{N}(\nu(U)) \equiv-N \bmod D .
$$


Quaternionic orders. Let $V(x)=U x^{\prime}$, and let

$$
R_{U}=(K \oplus K V) \cap \operatorname{End}(L) .
$$

Then $R_{U}$ is a Rosati-invariant order in the quaternion algebra generated by $V$. Clearly $\mathcal{O} \subset R_{U}$, so we can also regard $\left(R_{U}, *\right)$ as an involutive algebra over $\mathcal{O}$. We will show that $N=\operatorname{det}(U)$ and $\nu(U)$ determine $\left(R_{U}, *\right)$ up to isomorphism.

Models. We begin by constructing a model algebra $\left(R_{N}(\nu), *\right)$ over $\mathcal{O}_{D}$ for every $\nu \in \mathcal{O} / \mathcal{D}$ with $\mathrm{N}(\nu)=-N \neq 0 \bmod D$.

Let $Q_{N}=K \oplus K V$ be the abstract quaternion algebra with the relations $V^{2}=-N$ and $V k=k^{\prime} V$. Define an involution on $Q_{N}$ by $\left(k_{1}+k_{2} V\right)^{*}=$ $\left(k_{1}-k_{2}^{\prime} V\right)$, and let $R_{N}(\nu)$ be the order in $Q_{N}$ defined by

$$
R_{N}(\nu)=\left\{\alpha+\beta V: \alpha, \beta \in \mathcal{O}^{\vee}, \alpha+\beta \nu \in \mathcal{O} .\right\}
$$

Note that $\mathcal{O}^{\vee} \cdot \mathcal{D} \subset \mathcal{O}$, so the definition of $R_{N}(\nu)$ depends only on the class of $\nu$ in $\mathcal{O} / \mathcal{D}$. To check that $R_{N}(\nu)$ is an order, note that

$$
(\alpha+\beta V)(\gamma+\delta V)=(\kappa+\lambda V)=\left(\alpha \gamma-N \beta \delta^{\prime}\right)+\left(\alpha \delta+\beta \gamma^{\prime}\right) V
$$

since $-N \equiv \mathrm{N}(\nu)=\nu \nu^{\prime} \bmod D$, we have

$$
\begin{aligned}
\kappa+\nu \lambda & \equiv\left(\alpha \gamma+\nu \nu^{\prime} \beta \delta^{\prime}\right)+\nu\left(\alpha \delta+\beta \gamma^{\prime}\right) \\
& =(\alpha+\beta \nu)\left(\gamma^{\prime}+\delta^{\prime} \nu^{\prime}\right)+\alpha\left(\gamma-\gamma^{\prime}+\nu \delta-\nu^{\prime} \delta^{\prime}\right) \\
& \equiv 0+0 \bmod \mathcal{O}
\end{aligned}
$$

and thus $R_{U}$ is closed under multiplication.

Theorem 2.6 The quaternionic order $R_{N}(\nu)$ has discriminant $N^{2}$.

Proof. Note that the inclusions

$$
\mathcal{O} \oplus \mathcal{O} V \subset R_{N}(\nu) \subset \mathcal{O}^{\vee} \oplus \mathcal{O}^{\vee} V
$$

each have index $D$. The quaternionic order $\mathcal{O} \oplus \mathcal{O} V$ has discriminant $D^{2} N^{2}$, since $V^{2}=-N$ and $\operatorname{Tr} \mid \mathcal{O} V=0$, and thus $R_{N}(\nu)$ has discriminant $N^{2}$. 
Theorem 2.7 We have $\left(R_{N}(\nu), *\right) \cong\left(R_{M}(\mu), *\right)$ iff $N=M$ and $\nu= \pm \mu$.

Proof. The element $V \in R_{N}(\nu)$ is, up to sign, the order's unique primitive generator, in the sense that $V^{*}=-V, V k=k^{\prime} V$ for all $k \in \mathcal{O}_{D}, V^{2} \neq 0$, $k+D^{-1 / 2} V \in R_{N}(\nu)$ for some $k \in K$, and $V$ is not a proper multiple of another element in $R_{N}(\nu)$ with the same properties. Thus the structure of $\left(R_{N}(\nu), *\right)$ as an $\mathcal{O}_{D}$-algebra determines $V \in R_{N}(\nu)$ up to sign, and $V$ determines $N=-V^{2}$ and the constant $\nu \in \mathcal{O} / \mathcal{D}$ in the relation $\alpha+\beta \nu \in \mathcal{O}$ defining $R_{N}(\nu) \subset K \oplus K V$.

Theorem 2.8 If $U$ is a primitive generator matrix, then we have

$$
\left(R_{U}, *\right) \cong\left(R_{N}(\nu), *\right)
$$

where $N=\operatorname{det}(U)$ and $\nu=\nu(U)$.

Proof. Setting $V(x)=U x^{\prime}$, we need only verify that $(K \oplus K V) \cap \operatorname{End}(L)$ coincides with the order $R_{N}(\nu)$ defined by (2.7). To see this, let

$$
T(x)=\alpha x+\beta V(x)=\alpha x+\beta\left(\begin{array}{cc}
\mu & b D \\
-a & -\mu^{\prime}
\end{array}\right) x^{\prime}
$$

in $K \oplus K V$. By Corollary 2.2, $T$ is integral iff

(i) $a \beta, b \beta, \mu \beta, \mu^{\prime} \beta \in \mathcal{O}^{\vee}$,

(ii) $\alpha \in \mathcal{O}^{\vee}$,

(iii) $\alpha+\beta \mu \in \mathcal{O}$ and

(iv) $\alpha+\beta \mu^{\prime} \in \mathcal{O}$.

Using (iii), condition (iv) can be replaced by

(iv') $\beta\left(\mu-\mu^{\prime}\right) / \sqrt{D} \in \mathcal{O}^{\vee}$.

Since $U$ is primitive, the ideal $\left(a, b, \mu,\left(\mu-\mu^{\prime}\right) / \sqrt{D}\right)$ is equal to $\mathcal{O}$. Thus (i) and $\left(\mathrm{iv}^{\prime}\right)$ together are equivalent to the condition $\beta \in \mathcal{O}^{\vee}$, and we are left with the definition of $R_{N}(\nu)$. 
Remark. In general, the invariants $\operatorname{det}(U)$ and $\nu(U)$ do not determine the embedding $R_{U} \subset \operatorname{End}(L)$ up to conjugacy. For example, when $D$ is odd, the generator matrices $U_{1}=\left(\begin{array}{cc}0 & D^{2} \\ -D & 0\end{array}\right)$ and $U_{2}=\left(\begin{array}{cc}0 & D^{3} \\ -1 & 0\end{array}\right)$ have the same invariants, but the corresponding endomorphisms are not conjugate in $\operatorname{End}(L)$ because

$$
L / V_{1}(L) \cong\left(\mathbb{Z} / D \times \mathbb{Z} / D^{2}\right)^{2}
$$

while

$$
L / V_{2}(L) \cong \mathbb{Z} / D \times \mathbb{Z} / D^{2} \times \mathbb{Z} / D^{3} .
$$

Extra quadratic orders. Finally we determine when the algebra $R_{N}(\nu)$ contains a second, independent quadratic order $\mathcal{O}_{E}$.

Theorem 2.9 The algebra $\left(R_{N}(\nu), *\right)$ contains a self-adjoint element $T \notin$ $\mathcal{O}_{D}$ generating a copy of $\mathcal{O}_{E}$ iff there exist $e, \ell \in \mathbb{Z}$ such that

$$
E D=e^{2}+4 N \ell^{2}, \quad \ell \neq 0
$$

and $(e+E \sqrt{D}) / 2+\ell \nu=0 \bmod \mathcal{D}$.

Proof. Given $e, \ell$ as above, let

$$
T=\alpha+\beta V=D^{-1 / 2}\left(\frac{e+E \sqrt{D}}{2}+\ell V\right) .
$$

Then we have $T=T^{*}, T \in R_{N}(\nu)$ and $T^{2}-e T+\left(E-E^{2}\right) / 4=0$; therefore $\mathbb{Z}[T] \cong \mathcal{O}_{E}$. A straightforward computation shows that, conversely, any independent copy of $\mathcal{O}_{E}$ in $R_{N}(\nu)$ arises as above.

For additional background on quaternion algebras, see e.g. [Vi], [MR] and $[\mathrm{Mn}]$.

\section{Modular curves and surfaces}

In this section we describe modular curves on Hilbert modular surfaces from the perspective of the Abelian varieties they determine.

Abelian varieties. A principally polarized Abelian variety is a complex torus $A \cong \mathbb{C}^{g} / L$ equipped with a unimodular symplectic form $\langle x, y\rangle$ on $L \cong \mathbb{Z}^{2 g}$, whose extension to $L \otimes \mathbb{R} \cong \mathbb{C}^{g}$ satisfies

$$
\langle x, y\rangle=\langle i x, i y\rangle \quad \text { and } \quad\langle x, i x\rangle \geq 0 .
$$


The ring $\operatorname{End}(A)=\operatorname{End}(L) \cap \operatorname{End}\left(\mathbb{C}^{g}\right)$ is Rosati invariant, and coincides with the endomorphism ring of $A$ as a complex Lie group. We have $\operatorname{Tr}\left(T T^{*}\right) \geq 0$ for all $T \in \operatorname{End}(A)$.

Every Abelian variety can be presented in the form

$$
A=\mathbb{C}^{g} /\left(\mathbb{Z}^{g} \oplus \Pi \mathbb{Z}^{g}\right),
$$

where $\Pi$ is an element of the Siegel upper halfplane

$$
\mathfrak{H}_{g}=\left\{\Pi \in M_{g}(\mathbb{C}): \Pi^{t}=\Pi \text { and } \operatorname{Im}(\Pi) \text { is positive-definite }\right\} .
$$

The symplectic form on $L=\mathbb{Z}^{g} \oplus \Pi \mathbb{Z}^{g}$ is given by $\left(\begin{array}{cc}0 & I \\ -I & 0\end{array}\right)$. Any two such presentations of $A$ differ by an automorphism of $L$, so the moduli space of abelian varieties of genus $g$ is given by the quotient space

$$
\mathcal{A}_{g}=\mathfrak{H}_{g} / \mathrm{Sp}_{2 g}(\mathbb{Z}) \text {. }
$$

Real multiplication. As in $\S 2$, let $D>0$ be the discriminant of a real quadratic order $\mathcal{O}_{D}$, and let $K=\mathcal{O} \otimes \mathbb{Q}$. Fix a real place $\iota_{1}: K \rightarrow \mathbb{R}$, and set $\iota_{2}(k)=\iota_{1}\left(k^{\prime}\right)$.

We will regard $K$ as a subfield of the reals, using the fixed embedding $\iota_{1}: K \subset \mathbb{R}$. The case $D=d^{2}$ is treated with the understanding that the real numbers $\left(k, k^{\prime}\right)$ implicitly denote $\left(\iota_{1}(k), \iota_{2}(k)\right), k \in K$.

An Abelian variety $A \in \mathcal{A}_{2}$ admits real multiplication by $\mathcal{O}_{D}$ if there is a self-adjoint endomorphism $T \in \operatorname{End}(A)$ generating a proper action of $\mathbb{Z}[T] \cong \mathcal{O}_{D}$ on $A$. Any such variety can be presented in the form

$$
A_{\tau}=\mathbb{C}^{2} /\left(\mathcal{O}_{D} \oplus \mathcal{O}_{D}^{\vee} \tau\right)=\mathbb{C}^{2} / \phi_{\tau}(L)
$$

where $\tau=\left(\tau_{1}, \tau_{2}\right) \in \mathbb{H} \times \mathbb{H}$ and where $L=\mathcal{O} \oplus \mathcal{O}^{\vee}$ is embedded in $\mathbb{C}^{2}$ by the map

$$
\phi_{\tau}\left(x_{1}, x_{2}\right)=\left(x_{1}+x_{2} \tau_{1}, x_{1}^{\prime}+x_{2}^{\prime} \tau_{2}\right) .
$$

As in $\S 2$, the symplectic form on $L$ is given by $\langle x, y\rangle=\operatorname{Tr}_{\mathbb{Q}}^{K}(x \wedge y)$, and the action of $\mathcal{O}_{D}$ on $\mathbb{C}^{2} \supset L$ is given simply by $k \cdot\left(z_{1}, z_{2}\right)=\left(k z_{1}, k^{\prime} z_{2}\right)$.

Eigenforms. The Abelian variety $A_{\tau}$ comes equipped with a distinguished pair of normalized eigenforms $\eta_{1}, \eta_{2} \in \Omega\left(A_{\tau}\right)$. Using the isomorphism $H_{1}\left(A_{\tau}, \mathbb{Z}\right) \cong L$, these forms are characterized by the property that

$$
\phi_{\tau}(C)=\left(\int_{C} \eta_{1}, \int_{C} \eta_{2}\right) .
$$


Modular surfaces. If we change the identification $L \cong H_{1}\left(A_{\tau}, \mathbb{Z}\right)$ by an automorphism $g$ of $L$, we obtain an isomorphic Abelian variety $A_{g \cdot \tau}$. Thus the moduli space of Abelian varieties with real multiplication by $\mathcal{O}_{D}$ is given by the Hilbert modular surface

$$
X_{D}=(\mathbb{H} \times \mathbb{H}) / \mathrm{SL}\left(\mathcal{O}_{D} \oplus \mathcal{O}_{D}^{\vee}\right) .
$$

The point $g(\tau)$ is characterized by the property that

$$
\phi_{g \cdot \tau}=\chi(g, \tau) \phi_{\tau} \circ g^{-1}
$$

for some matrix $\chi(g, \tau) \in \mathrm{GL}_{2}(\mathbb{C})$; explicitly, we have

$$
\left(\begin{array}{ll}
a & b \\
c & d
\end{array}\right) \cdot\left(\tau_{1}, \tau_{2}\right)=\left(\frac{a \tau_{1}-b}{-c \tau_{1}+d}, \frac{a^{\prime} \tau_{2}-b^{\prime}}{-c^{\prime} \tau_{2}+d^{\prime}}\right)
$$

and

$$
\chi(g, \tau)=\left(\begin{array}{cc}
\left(d-c \tau_{1}\right)^{-1} & 0 \\
0 & \left(d^{\prime}-c^{\prime} \tau_{2}\right)^{-1}
\end{array}\right) .
$$

A point $[\tau] \in X_{D}$ gives an Abelian variety $\left[A_{\tau}\right] \in \mathcal{A}_{2}$ with a chosen embed$\operatorname{ding} \mathcal{O}_{D} \rightarrow \operatorname{End}\left(A_{\tau}\right)$. Similarly, a point $\tau \in \widetilde{X}_{D}=\mathbb{H} \times \mathbb{H}$ gives an Abelian variety with a distinguished isomorphism or marking, $L \cong H_{1}\left(A_{\tau}, \mathbb{Z}\right)$, sending $\mathcal{O}_{D}$ into $\operatorname{End}\left(A_{\tau}\right)$.

Modular embedding. The modular embedding

$$
p_{D}: X_{D} \rightarrow \mathcal{A}_{2}
$$

is given by $[\tau] \mapsto\left[A_{\tau}\right]$. To write $p_{D}$ explicitly, note that the embedding $\phi_{\tau}: L \rightarrow \mathbb{C}^{2}$ can be expressed with respect to the basis $\left(a_{1}, a_{2}, b_{1}, b_{2}\right)$ for $L$ given in (2.2) by the matrix

$$
\phi_{\tau}=\left(\begin{array}{cccc}
1 & \gamma & -\tau_{1} \gamma^{\prime} / \sqrt{D} & \tau_{1} / \sqrt{D} \\
1 & \gamma^{\prime} & \tau_{2} \gamma / \sqrt{D} & -\tau_{2} / \sqrt{D}
\end{array}\right)=(A, B) .
$$

Consequently we have $A_{\tau} \cong \mathbb{C}^{2} /\left(\mathbb{Z}^{2} \oplus \Pi \mathbb{Z}^{2}\right)$, where

$$
\Pi=\widetilde{p_{D}}(\tau)=A^{-1} B=\frac{1}{D}\left(\begin{array}{cc}
\tau_{1}\left(\gamma^{\prime}\right)^{2}+\tau_{2} \gamma^{2} & -\tau_{1} \gamma^{\prime}-\tau_{2} \gamma \\
-\tau_{1} \gamma^{\prime}-\tau_{2} \gamma & \tau_{1}+\tau_{2}
\end{array}\right)
$$

The map $X_{D} \rightarrow p_{D}\left(X_{D}\right)$ has degree two. 
Modular curves. Given a matrix $U(x)=\left(\begin{array}{ll}a & b \\ c & d\end{array}\right) \in M_{2}(K) \cap \operatorname{End}(L) \operatorname{such}$ that $U^{\prime}=-U^{*}$, let $V(x)=U x^{\prime}$ and define

$$
\mathbb{H}_{U}=\left\{\tau \in \mathbb{H} \times \mathbb{H}: V \in \operatorname{End}\left(A_{\tau}\right)\right\} .
$$

It is straightforward to check that

$$
\mathbb{H}_{U}=\left\{\left(\tau_{1}, \tau_{2}\right): \tau_{2}=\frac{d \tau_{1}+b}{c \tau_{1}+a}\right\} ;
$$

indeed, when $\tau_{1}$ and $\tau_{2}$ are related as above, the map $\phi_{\tau}: L \rightarrow \mathbb{C}^{2}$ satisfies

$$
\phi_{\tau}(V(x))=\left(\begin{array}{cc}
0 & a+c \tau_{1} \\
a^{\prime}+c^{\prime} \tau_{2} & 0
\end{array}\right) \phi_{\tau}(x),
$$

exhibiting the complex-linearity of $V$. Note that $\mathbb{H}_{U}=\emptyset$ if $\operatorname{det}(U)<0$.

We now restrict attention to the case where $U$ is a generator matrix. Then by the results of $\S 2$, we have:

Theorem 3.1 The ring $\operatorname{End}\left(A_{\tau}\right)$ contains a quaternionic order extending $\mathcal{O}_{D}$ if and only if $\tau \in \mathbb{H}_{U}$ for some generator matrix $U$.

Let $F_{U} \subset X_{D}$ denote the projection of $\mathbb{H}_{U}$ to the quotient $(\mathbb{H} \times \mathbb{H}) / \operatorname{SL}\left(\mathcal{O}_{D} \oplus \mathcal{O}_{D}^{\vee}\right)$. Following [Hir, §5.3], we define the modular curve $F_{N}$ by

$F_{N}=\bigcup\left\{F_{U}: U\right.$ is a primitive generator matrix with $\left.\operatorname{det}(U)=N.\right\}$.

It can be shown that $F_{N}$ is an algebraic curve on $X_{D}$.

To describe this curve more precisely, let

$$
F_{N}(\nu)=\left\{F_{U}: U \text { is primitive, } \operatorname{det}(U)=N \text { and } \nu(U)= \pm \nu\right\},
$$

where $\nu \in \mathcal{O}_{D} / \mathcal{D}_{D}$. Note that we have

$$
F_{N}(\nu) \neq \emptyset \Longleftrightarrow N(\nu)=-N \bmod D
$$

by equation (2.6), $F_{N}(\nu)=F_{N}(-\nu)$, and $F_{N}=\bigcup F_{N}(\nu)$.

The results of $\S 2$ give the structure of the quaternion ring generated by $V(x)=U x^{\prime}$.

Theorem 3.2 The curve $F_{N}(\nu) \subset X_{D}$ coincides with the locus of Abelian varieties such that

$$
\mathcal{O}_{D} \subset R \subset \operatorname{End}\left(A_{\tau}\right),
$$

for some properly embedded quaternionic order $(R, *)$ isomorphic to $\left(R_{N}(\nu), *\right)$. 
Corollary 3.3 The curve $F_{N}$ is the locus where $\mathcal{O}_{D} \subset \operatorname{End}\left(A_{\tau}\right)$ extends to a properly embedded, Rosati-invariant quaternionic order of discriminant $N^{2}$.

Two quadratic orders. We can now describe the locus $X_{D}(E)$ of Abelian varieties with an independent, self-adjoint action of $\mathcal{O}_{E}$. (We do not require the action of $\mathcal{O}_{E}$ to be proper.)

To state this description, it is useful to define:

$$
T_{N}=\bigcup\left\{F_{U}: \operatorname{det}(U)=N\right\}=\bigcup F_{N / \ell^{2}},
$$

and

$$
T_{N}(\nu)=\bigcup\left\{F_{U}: \operatorname{det}(U)=N, \nu(U)= \pm \nu\right\} .
$$

Then Theorem 2.9 implies:

Theorem 3.4 The locus $X_{D}(E)$ is given by

$$
X_{D}(E)=\bigcup T_{N}((e+E \sqrt{D}) / 2),
$$

where the union is over all $N>0$ and $e \in \mathbb{Z}$ such that $E D=e^{2}+4 N$.

Corollary 3.5 We have $X_{D}(1)=\bigcup\left\{T_{N}((e+\sqrt{D}) / 2): e^{2}+4 N=D\right\}$.

Refined modular curves. To conclude we show that in general the expression $F_{N}=\bigcup F_{N}(\nu)$ gives a proper refinement of $F_{N}$. First note:

Theorem 3.6 We have $F_{N}(\nu)=F_{N}$ iff $\pm \nu$ are the only solutions to

$$
N(\xi)=-N \bmod D, \quad \xi \in \mathcal{O}_{D} / \mathcal{D}_{D} .
$$

Corollary 3.7 If $D=p$ is prime, then $F_{N}=F_{N}(\nu)$ whenever $F_{N}(\nu) \neq \emptyset$.

Proof. In this case, according to (2.1), the norm map

$$
N: \mathcal{O}_{D} / \mathcal{D}_{D} \stackrel{\operatorname{Tr}}{\cong} \mathbb{Z} / p \rightarrow \mathbb{Z} / p
$$

is given by $N(\xi)=\xi^{2} / 4$. Since $F_{N}(\nu) \neq \emptyset$, we have $N(\nu)=-N$; and since $\mathbb{Z} / p$ is a field, $\pm \nu$ are the only solutions to this equation. 
Corollary 3.8 When $D$ is prime, we have $X_{D}(E)=\bigcup T_{\left(E D-e^{2}\right) / 4}$.

Now consider the case $D=21$, the first odd discriminant which is not a prime. Then the norm map is still given by $N(\xi)=\xi^{2} / 4$ on $\mathcal{O}_{D} / \mathcal{D}_{D} \cong \mathbb{Z} / D$, but now $\mathbb{Z} / D$ is not a field. For example, the equation $\xi^{2}=1 \bmod D$ has four solutions, namely $\xi=1,8,13$ or 20 . These give four solutions to the equation $N(\xi)=-5$, and hence contribute two distinct terms to the expression

$$
F_{5}=\bigcup F_{5}(\nu)=F_{5}((1+\sqrt{21}) / 2) \cup F_{5}((8+\sqrt{21}) / 2) .
$$

Only one of these terms appears in the expression for $X_{D}(1)$. In fact, since $21=1^{2}+4 \cdot 5=3^{2}+4 \cdot 3$, by Corollary 3.5 we have

$$
\begin{aligned}
X_{21}(1) & =F_{3} \cup F_{5}((1+\sqrt{21}) / 2) \\
& \neq F_{3} \cup F_{5} .
\end{aligned}
$$

(The full curve $F_{3}$ appears because the only solutions to $N(\xi)=\xi^{2} / 4=$ $-3 \bmod 21$ are $\xi= \pm 3$.)

Using Theorem 3.6, it is similarly straightforward to check other small discriminants; for example:

Theorem 3.9 For $D \leq 30$ we have $X_{D}(1)=\bigcup_{e^{2}+4 N=D} T_{N}$ when $D=$ $4,5,8,9,13,17,25$ and 29 , but not when $D=12,16,20,21,24$ or 28 .

Notes. For more background on modular curves and surfaces, see [Hir], [HZ2], [HZ1], [BL], [Mc7, §4] and [vG]. Our $U=\left(\begin{array}{cc}\mu & b D \\ -a & -\mu^{\prime}\end{array}\right)$ corresponds to the skew-Hermitian matrix $B=\sqrt{D}\left(\begin{array}{cc}a & \mu \\ \mu^{\prime} & b D\end{array}\right)$ in [vG, Ch. V]. Note that (3.3) agrees with the standard action $(a \tau+b) /(c \tau+d)$ up to the automorphism $\left(\begin{array}{ll}a & b \\ c & d\end{array}\right) \mapsto\left(\begin{array}{cc}a & -b \\ -c & d\end{array}\right)$ of $\mathrm{SL}_{2}(K)$. We remark that $X_{D}$ can also be presented as the quotient $(\mathbb{H} \times-\mathbb{H}) / \mathrm{SL}_{2}\left(\mathcal{O}_{D}\right)$, using the fact that $\sqrt{D}^{\prime}=-\sqrt{D}$; on the other hand, the surfaces $(\mathbb{H} \times \mathbb{H}) / \mathrm{SL}_{2}\left(\mathcal{O}_{D}\right)$ and $X_{D}$ are generally not isomorphic (see e.g. [HH].)

It is known that the intersection numbers $\left\langle T_{N}, T_{M}\right\rangle$ form the coefficients of a modular form [HZ1], [vG, Ch. VI]. The results of [GKZ] suggest that the intersection numbers of the refined modular curves $T_{N}(\nu)$ may similarly yield a Jacobi form.

\section{Laminations}

In this section we show algebraically that $\widetilde{X}_{D}(1)$ gives a lamination of $\mathbb{H} \times$ $\mathbb{H}$ by countably many disjoint hyperbolic planes. We also describe these 
laminations explicitly for small values of $D$. Another proof of laminarity appears in $\S 7$.

Jacobian varieties. Let $\Omega(X)$ denote the space of holomorphic 1-forms on a compact Riemann surface $X$. The Jacobian of $X$ is the Abelian variety $\operatorname{Jac}(X)=\Omega(X)^{*} / H_{1}(X, \mathbb{Z})$, polarized by the intersection pairing on 1-cycles.

In the case of genus two, any principally polarized Abelian variety $A$ is either a Jacobian or a product of polarized elliptic curves. The latter case occurs iff $A$ admits real multiplication by $\mathcal{O}_{1}$, generated by projection to one of the factors of $A \cong B_{1} \times B_{2}$. In particular, we have:

Theorem 4.1 For any $D \geq 4$, the locus of Jacobian varieties in $X_{D}$ is given by $X_{D}-X_{D}(1)$.

Laminations. To describe $X_{D}(1)$ in more detail, given $N>0$ such that $D=e^{2}+4 N$ let

$$
\begin{aligned}
\Lambda_{D}^{N}= & \left\{U \in M_{2}(K): U \text { is a generator matrix, } \operatorname{det}(U)=N\right. \text { and } \\
& \left.\nu(U) \equiv \pm(e+\sqrt{D}) / 2 \bmod \mathcal{D}_{D}\right\},
\end{aligned}
$$

and let $\Lambda_{D}$ be the union of all such $\Lambda_{D}^{N}$. Note that if $U$ is in $\Lambda_{D}$, then $-U, U^{\prime}$ and $U^{*}$ are also in $\Lambda_{D}$.

By Corollary 3.5, the preimage of $X_{D}(1)$ in $\widetilde{X}_{D}=\mathbb{H} \times \mathbb{H}$ is given by:

$$
\widetilde{X}_{D}(1)=\bigcup\left\{\mathbb{H}_{U}: U \in \Lambda_{D}\right\}
$$

Note that each $\mathbb{H}_{U}$ is the graph of a Möbius transformation.

Theorem 4.2 The locus $\widetilde{X}_{D}(1)$ gives a lamination of $\mathbb{H} \times \mathbb{H}$ by countably many hyperbolic planes.

(This means any two planes in $\tilde{X}_{D}(1)$ are either identical or disjoint.)

For the proof, it suffices to show that the difference $g \circ h^{-1}$ of two Möbius transformations in $\Lambda_{D}$ is never elliptic. Since $\Lambda_{D}$ is invariant under $U \mapsto$ $U^{*}=(\operatorname{det} U) U^{-1}$, this in turn follows from:

Theorem 4.3 For any $U_{1}, U_{2} \in \Lambda_{D}$, we have $\operatorname{Tr}\left(U_{1} U_{2}\right)^{2} \geq 4 \operatorname{det}\left(U_{1} U_{2}\right)$.

Proof. By the definition of $\Lambda_{D}$, we can write $D=e_{i}^{2}+4 \operatorname{det}\left(U_{i}\right)=e_{i}^{2}+4 N_{i}$, where $e_{i} \geq 0$. We can also assume that

$$
U_{i}=\left(\begin{array}{cc}
\mu_{i} & b_{i} D \\
-a_{i} & -\mu_{i}^{\prime}
\end{array}\right)
$$


satisfies

$$
\mu_{i} \equiv\left(x_{i}+y_{i} \sqrt{D}\right) / 2 \equiv\left(e_{i}+\sqrt{D}\right) / 2 \bmod \mathcal{D}_{D}
$$

(replacing $U_{i}$ with $-U_{i}$ if necessary). It follows that $y_{i}$ is odd and $x_{i}=$ $e_{i} \bmod D$, which implies

$$
\operatorname{Tr}\left(U_{1} U_{2}\right) \equiv \operatorname{Tr}\left(\mu_{1} \mu_{2}\right)=\left(x_{1} x_{2}+D y_{1} y_{2}\right) / 2 \equiv\left(e_{1} e_{2}-D\right) / 2 \bmod D .
$$

(The factor of $1 / 2$ presents no difficulties, because $x_{i}$ is even when $D$ is even.)

Now suppose

$$
\operatorname{Tr}\left(U_{1} U_{2}\right)^{2}<4 \operatorname{det}\left(U_{1} U_{2}\right)=4 N_{1} N_{2} .
$$

Then we have $\left|\operatorname{Tr}\left(U_{1} U_{2}\right)\right|<2 \sqrt{N_{1} N_{2}} \leq D / 2$, and thus (4.1) implies

$$
\operatorname{Tr}\left(U_{1} U_{2}\right)=\left(e_{1} e_{2}-D\right) / 2 \text {. }
$$

But this implies

$$
\begin{aligned}
4 \operatorname{Tr}\left(U_{1} U_{2}\right)^{2} & =\left(D-e_{1} e_{2}\right)^{2} \\
& \geq\left(D-e_{1}^{2}\right)\left(D-e_{2}^{2}\right)=\left(4 N_{1}\right)\left(4 N_{2}\right)=16 \operatorname{det}\left(U_{1} U_{2}\right),
\end{aligned}
$$

contradicting (4.2).

Small discriminants. To conclude we record a few cases where $\Lambda_{D}$ admits a particularly economical description.

For concreteness, we will present $\Lambda_{D}$ as a set matrices in $\mathrm{GL}_{2}^{+}(\mathbb{R})$ using the chosen real place $\iota_{1}: K \rightarrow \mathbb{R}$. This works even when $D=d^{2}$, since both $\mu$ and $\mu^{\prime}$ appear on the diagonal of $U \in \Lambda_{D}$ (no information is lost). Under the standard action $\left(\begin{array}{ll}a & b \\ c & d\end{array}\right) \cdot z=(a z+b) /(c z+d)$ of $\mathrm{GL}_{2}^{+}(\mathbb{R})$ on $\mathbb{H}$, we can then write

$$
\tilde{X}_{D}(1)=\bigcup_{\Lambda_{D}}\left\{\left(\tau_{1}, \tau_{2}\right): \tau_{2}=U\left(\tau_{1}\right)\right\}
$$

This holds despite the twist in the definition (3.5) of $\mathbb{H}_{U}$, because $\Lambda_{D}$ is invariant under $\left(\begin{array}{ll}a & b \\ c & d\end{array}\right) \mapsto\left(\begin{array}{ll}d & b \\ c & a\end{array}\right)$.

Theorem 4.4 For $D=4,5,8,9$ and 13 respectively, we have:

$$
\begin{aligned}
\Lambda_{4} & =\left\{U \in \mathrm{M}_{2}(\mathbb{Z}): \operatorname{det}(U)=1 \text { and } U \equiv\left(\begin{array}{l}
* 0 \\
* *
\end{array}\right) \bmod 4\right\}, \\
\Lambda_{5} & =\left\{U=\left(\begin{array}{cc}
\mu & b D \\
-a & -\mu^{\prime}
\end{array}\right): \operatorname{det}(U)=1\right\}, \\
\Lambda_{8} & =\Lambda_{8}^{1} \cup \Lambda_{8}^{2}=\left\{U=\left(\begin{array}{cc}
\mu & b D \\
-a-\mu^{\prime}
\end{array}\right): \operatorname{det}(U)=1 \text { or } 2\right\}, \\
\Lambda_{9} & =\left\{U \in \mathrm{M}_{2}(\mathbb{Z}): \operatorname{det}(U)=2 \text { and } U \equiv\left(\begin{array}{cc}
* 0 \\
* *
\end{array}\right) \bmod 9\right\}, \quad \text { and } \\
\Lambda_{13} & =\Lambda_{13}^{1} \cup \Lambda_{13}^{3}=\left\{U=\left(\begin{array}{cc}
\mu & b D \\
-a-\mu^{\prime}
\end{array}\right): \operatorname{det}(U)=1 \text { or } 3\right\},
\end{aligned}
$$


where it is understood that $a, b \in \mathbb{Z}$ and $\mu \in \mathcal{O}_{D}$.

Proof. Recall from Theorem 3.9 that $X_{D}(1)=\bigcup_{e^{2}+4 N=D} T_{N}$ when $D=$ $4,5,8,9$ and 13 . When this equality holds, we can ignore the condition on $\nu(U)$ in the definition of $\Lambda_{D}$. The cases $D=5,8$ and 13 then follow directly from the definition of $\Lambda_{D}^{N}$. For $D=9$, we note that any integral matrix satisfying $\operatorname{det}\left(\begin{array}{cc}x & 9 b \\ -a & y\end{array}\right)=2$ also satisfies $x+y=0 \bmod 3$, and thus it can be written in the form $\left(\begin{array}{cc}\mu & b D \\ -a & -\mu^{\prime}\end{array}\right)$ with

$$
\mu=\frac{(x-y)+(x+y) \sqrt{9} / 3}{2} .
$$

Similar considerations apply when $D=4$.

\section{Foliations of Teichmüller space}

In this section we introduce a family of foliations $\mathcal{F}_{i}$ of Teichmüller space, related to normalized Abelian differentials and their periods $\tau_{i j}=\int_{b_{i}} \omega_{j}$. We then show:

Theorem 5.1 There is a unique holomorphic section of the period map

$$
\tau_{i i}: \mathcal{T}_{g} \rightarrow \mathbb{H}
$$

through any $Y \in \mathcal{T}_{g}$. Its image is the leaf of $\mathcal{F}_{i}$ containing $Y$.

The case $g=2$ will furnish the desired foliations of Hilbert modular surfaces.

Abelian differentials. Let $Z_{g}$ be a smooth oriented surface of genus $g$. Let $\mathcal{T}_{g}$ be the Teichmüller space of Riemann surfaces $Y$, each equipped with an isotopy class of homeomorphism or marking $Z_{g} \rightarrow Y$. The marking determines a natural identification between $H_{1}\left(Z_{g}\right)$ and $H_{1}(Y)$ used frequently below.

Let $\Omega \mathcal{T}_{g} \rightarrow \mathcal{T}_{g}$ denote the bundle of nonzero Abelian differentials $(Y, \omega)$, $\omega \in \Omega(Y)$. For each such form we have a period map

$$
I(\omega): H_{1}\left(Z_{g}, \mathbb{Z}\right) \rightarrow \mathbb{C}
$$

given by $I(\omega): C \rightarrow \int_{C} \omega$. There is a natural action of $\mathrm{GL}_{2}^{+}(\mathbb{R})$ on $\Omega \mathcal{T}_{g}$, satisfying

$$
I(A \cdot \omega)=A \circ I(\omega)
$$


under the identification $\mathbb{C}=\mathbb{R}^{2}$ given by $x+i y=(x, y)$.

Each orbit $\mathrm{GL}_{2}^{+}(\mathbb{R}) \cdot(Y, \omega)$ projects to a complex geodesic

$$
f: \mathbb{H} \rightarrow \mathcal{T}_{g},
$$

which can be normalized so that $f(i)=Y$ and

$$
\nu=\left.\frac{d f}{d t}\right|_{t=i}=\frac{i}{2} \frac{\bar{\omega}}{\omega}
$$

The subspace of $H^{1}\left(Z_{g}, \mathbb{R}\right)$ spanned by $(\operatorname{Re} \omega, \operatorname{Im} \omega)$ is constant along each orbit (cf. [Mc7, §3]).

Symplectic framings. Now let $\left(a_{1}, \ldots, a_{g}, b_{1}, \ldots, b_{g}\right)$ be a real symplectic basis for $H_{1}\left(Z_{g}, \mathbb{R}\right)$ (with $\left\langle a_{i}, b_{i}\right\rangle=-\left\langle b_{i}, a_{i}\right\rangle=1$ and all other products zero). Then for each $Y \in \mathcal{T}_{g}$, there exists a unique basis $\left(\omega_{1}, \ldots, \omega_{g}\right)$ of $\Omega(Y)$ such that $\int_{a_{i}} \omega_{j}=\delta_{i j}$. The period matrix

$$
\tau_{i j}(Y)=\int_{b_{i}} \omega_{j}
$$

then determines an embedding

$$
\tau: \mathcal{T}_{g} \rightarrow \mathfrak{H}_{g}
$$

This agrees with the usual Torelli embedding, up to composition with an element of $\operatorname{Sp}_{2 g}(\mathbb{R})$. Note that $\operatorname{Im}\left(\tau_{i i}(Y)\right)>0$ since $\operatorname{Im} \tau$ is positive definite.

The normalized 1-forms $\left(\omega_{i}\right)$ give a splitting

$$
\Omega(Y)=\oplus_{1}^{g} \mathbb{C} \omega_{i}=\oplus_{1}^{g} F_{i}(Y),
$$

and corresponding subbundles $F_{i} \mathcal{T}_{g} \subset \Omega T_{g}$.

Complex subspaces. Let $\left(a_{i}^{*}, b_{i}^{*}\right)$ denote the dual basis for $H^{1}\left(Z_{g}, \mathbb{R}\right)$, and let $S_{i}$ be the span of $\left(a_{i}^{*}, b_{i}^{*}\right)$. It easy to check that the following conditions are equivalent:

1. $S_{i}$ is a complex subspace of $H^{1}(Y, \mathbb{R}) \cong \Omega(Y)$.

2. $S_{i}$ is spanned by $\left(\operatorname{Re} \omega_{i}, \operatorname{Im} \omega_{i}\right)$.

3. The period matrix $\tau(Y)$ satisfies $\tau_{i j}=0$ for all $j \neq i$. 
Let $\mathcal{T}_{g}\left(S_{i}\right) \subset \mathcal{T}_{g}$ denote the locus where these condition hold. Note that condition (3) defines a totally geodesic subset

$$
H_{i} \cong \mathbb{H} \times \mathfrak{H}_{g-1} \subset \mathfrak{H}_{g}
$$

such that $\mathcal{T}_{g}\left(S_{i}\right)=\tau^{-1}\left(H_{i}\right)$.

Foliations. Next we show that the complex geodesics generated by the forms $\left(Y, \omega_{i}\right)$ give a foliation of Teichmüller space.

Theorem 5.2 The sub-bundle $F_{i} \mathcal{T}_{g} \subset \Omega \mathcal{T}_{g}$ is invariant under the action of $\mathrm{GL}_{2}^{+}(\mathbb{R})$, as is its restriction to $\mathcal{T}_{g}\left(S_{i}\right)$.

Proof. The invariance of $F_{i} \mathcal{T}_{g}$ is immediate from (5.1). To handle the restriction to $\mathcal{T}_{g}\left(S_{i}\right)$, recall that the span $W$ of $\left(\operatorname{Re} \omega_{i}, \operatorname{Im} \omega_{i}\right)$ is constant along orbits; thus the condition $W=S_{i}$ characterizing $\mathcal{T}_{g}\left(S_{i}\right)$ is preserved by the action of $\mathrm{GL}_{2}^{+}(\mathbb{R})$.

Corollary 5.3 The foliation of $F_{i} \mathcal{T}_{g}$ by $\mathrm{GL}_{2}^{+}(\mathbb{R})$ orbits projects to a foliation $\mathcal{F}_{i}$ of $\mathcal{T}_{g}$ by complex geodesics.

Corollary 5.4 The locus $\mathcal{T}_{g}\left(S_{i}\right)$ is also foliated by $\mathcal{F}_{i}$ : any leaf meeting $\mathcal{T}_{g}\left(S_{i}\right)$ is entirely contained therein.

Proof of Theorem 5.1. The proof uses Ahlfors' variational formula [Ah] and follows the same lines as the proof of [Mc4, Thm. 4.2]; it is based on the fact that the leaves of $\mathcal{F}_{i}$ are the geodesics along which the periods of $\omega_{i}$ change most rapidly.

Let $s: \mathbb{H} \rightarrow \mathcal{T}_{g}$ be a holomorphic section of $\tau_{i i}$. Let $v \in \mathrm{TH}$ be a unit tangent vector with respect to the hyperbolic metric $\rho=|d z| /(2 \operatorname{Im} z)$ of constant curvature -4 , mapping to $D s(v) \in \mathrm{T}_{Y} \mathcal{T}_{g}$. By the equality of the Teichmüller and Kobayashi metrics [Gd, Ch. 7], $D s(v)$ is represented by a Beltrami differential $\nu=\nu(z) d \bar{z} / d z$ on $Y$ with $\|\nu\|_{\infty} \leq 1$. But $s$ is a section, so the composition

$$
\tau_{i i} \circ s: \mathbb{H} \rightarrow \mathbb{H}
$$

is the identity; thus the norm of its derivative, given by Ahlfors' formula as

$$
\left\|D\left(\tau_{i i} \circ s\right)(\nu)\right\|=\left|\int_{Y} \omega_{i}^{2} \nu\right| / \int_{Y}\left|\omega_{i}\right|^{2},
$$

is one. It follows that $\nu=\bar{\omega}_{i} / \omega_{i}$ up to a complex scalar of modulus one, and thus $D s(v)$ is tangent to the complex geodesic generated by $\left(Y, \omega_{i}\right)$. Equivalently, $s(\mathbb{H})$ is everywhere tangent to the foliation $\mathcal{F}_{i}$; therefore its image is the unique leaf through $Y$. 


\section{Genus two}

We can now obtain results on Hilbert modular surfaces by specializing to the case of genus two. In this section we will show:

Theorem 6.1 There is a unique holomorphic section of $\tau_{1}$ passing through any given point of $\mathbb{H} \times \mathbb{H}-\widetilde{X}_{D}(1)$.

Here $\tau_{1}: \mathbb{H} \times \mathbb{H} \rightarrow \mathbb{H}$ is simply projection onto the first factor. This result is a restatement of Theorem 1.2 ; as in $\S 1$, we assume $D \geq 4$.

Framings for real multiplication. Let $g=2$, and choose a symplectic isomorphism

$$
L=H_{1}\left(Z_{g}, \mathbb{Z}\right) \cong \mathcal{O}_{D} \oplus \mathcal{O}_{D}^{\vee}
$$

We then have an action of $\mathcal{O}_{D}$ on $H_{1}\left(Z_{g}, \mathbb{Z}\right)$, and the elements $\{a, b\}=$ $\{(1,0),(0,1)\}$ in $L$ give a distinguished basis for

$$
H_{1}\left(Z_{g}, \mathbb{Q}\right)=L \otimes \mathbb{Q} \cong K^{2}
$$

as a vector space over $K=\mathcal{O}_{D} \otimes \mathbb{Q}$. Using the two Galois conjugate embeddings $K \rightarrow \mathbb{R}$, we obtain an orthogonal splitting

$$
H_{1}\left(Z_{g}, \mathbb{R}\right)=L \otimes \mathbb{R}=V_{1} \oplus V_{2}
$$

such that $k \cdot\left(C_{1}, C_{2}\right)=\left(k C_{1}, k^{\prime} C_{2}\right)$. The projections $\left(a_{i}, b_{i}\right)$ of $a, b \in L$ to each summand yield bases for $V_{i}$, which taken together give a standard symplectic basis for $H_{1}\left(Z_{g}, \mathbb{R}\right)$. (Note that $\left(a_{i}, b_{i}\right)$ is generally not an integral sympletic basis; indeed, when $K$ is a field, the elements $\left(a_{i}, b_{i}\right)$ do not even lie in $H_{1}\left(Z_{g}, \mathbb{Q}\right)$.)

Let $S_{i}^{D} \subset H^{1}\left(Z_{g}, \mathbb{R}\right)$ be the span of the dual basis $a_{i}^{*}, b_{i}^{*}$.

Theorem 6.2 The ring $\mathcal{O}_{D} \subset \operatorname{End}(L)$ acts by real multiplication on $\operatorname{Jac}(Y)$ if and only if $Y \in \mathcal{T}_{g}\left(S_{1}^{D}\right)$.

Proof. Since $g=2$ we have $S_{2}^{D}=\left(S_{1}^{D}\right)^{\perp}$, and thus $\mathcal{T}_{g}\left(S_{1}^{D}\right)=\mathcal{T}_{g}\left(S_{2}^{D}\right)$. But Jac $(Y)$ has real multiplication iff $S_{1}^{D}$ and $S_{2}^{D}$ are complex subspaces of $H^{1}(Y, \mathbb{R}) \cong \Omega(Y)$ so the result follows. (Cf. [Mc4, Lemma 7.4].) 
Sections. Let $E_{D}=X_{D}-X_{D}(1)$ denote the space of Jacobians in $X_{D}$, and $\widetilde{E}_{D}=\mathbb{H} \times \mathbb{H}-\widetilde{X}_{D}(1)$ its preimage in the universal cover. (The notation comes from [Mc7, §4], where we consider the space of eigenforms $\Omega E_{D}$ as a closed, $\mathrm{GL}_{2}^{+}(\mathbb{R})$-invariant subset of $\Omega \mathcal{M}_{g}$.)

By the preceding result, the Jacobian of any $Y \in \mathcal{T}_{g}\left(S_{1}^{D}\right)$ is an Abelian variety with real multiplication. Moreover, the marking of $Y$ determines a marking

$$
L \cong H_{1}(Y, \mathbb{Z}) \cong H_{1}(\operatorname{Jac}(Y), \mathbb{Z})
$$

of its Jacobian, and thus a map

$$
\mathrm{Jac}: \mathcal{T}_{g}\left(S_{1}^{D}\right) \rightarrow \widetilde{E}_{D}=\widetilde{X}_{D}-\widetilde{X}_{D}(1) .
$$

The basis $\left(a_{i}, b_{i}\right)$ yields a pair of normalized forms $\omega_{1}, \omega_{2} \in \Omega(Y)$. Similarly, we have a pair of normalized eigenforms $\eta_{1}, \eta_{2} \in \Omega\left(A_{\tau}\right)$ for each $\tau \in \widetilde{X}_{D}$, characterized by (3.2). Under the identification $\Omega(Y)=\Omega(\operatorname{Jac}(Y))$, we find:

Theorem 6.3 The forms $\omega_{i}$ and $\eta_{i}$ are equal for any $Y \in \mathcal{T}_{g}\left(S_{1}^{D}\right)$. Thus $\operatorname{Jac}(Y)=A_{\left(\tau_{1}, \tau_{2}\right)}$, where

$$
\left(\begin{array}{cc}
\tau_{1} & 0 \\
0 & \tau_{2}
\end{array}\right)=\tau_{i j}(Y)=\left(\int_{b_{i}} \omega_{j}\right) .
$$

Proof. The period map $\phi_{\tau}: L \rightarrow \mathbb{C}^{2}$ for $A_{\tau}=\operatorname{Jac}(Y)$ is given by

$$
\phi_{\tau}(C)=\left(\int_{C} \eta_{1}, \int_{C} \eta_{2}\right)=\left(x_{1}+x_{2} \tau_{1}, x_{1}^{\prime}+x_{2}^{\prime} \tau_{2}\right)
$$

where $C=\left(x_{1}, x_{2}\right) \in \mathcal{O}_{D} \oplus \mathcal{O}_{D}^{\vee}$; in particular, we have

$$
\phi_{\tau}(a)=\phi_{\tau}(1,0)=(1,1) .
$$

Since $\phi_{\tau}$ diagonalizes the action of $K$, we also have

$$
\phi_{\tau}(C)=\left(\int_{C_{1}} \eta_{1}, \int_{C_{2}} \eta_{2}\right)
$$

for any $C=C_{1}+C_{2} \in L \otimes \mathbb{R}=V_{1} \oplus V_{2}$. Setting $C=a$, this implies $\phi_{\tau}\left(a_{1}\right)=(1,0)$ and $\phi_{\tau}\left(a_{2}\right)=(0,1)$; thus $\int_{a_{i}} \eta_{j}=\delta_{i j}$, and therefore $\eta_{i}=\omega_{i}$ for $i=1,2$. Similarly, we have

$$
\phi_{\tau}(b)=\left(\tau_{1}, \tau_{2}\right)=\left(\tau_{11}, \tau_{22}\right),
$$

which implies $Y$ and $A_{\tau}$ are related by (6.1). 
Corollary 6.4 We have a commutative diagram

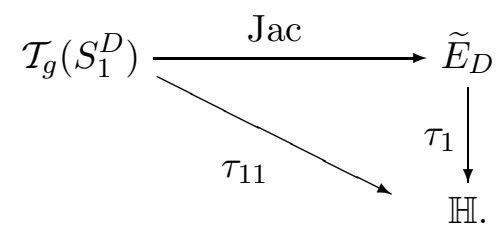

Proof of Theorem 6.1. Using the Torelli theorem, it follows easily that Jac $: \mathcal{T}_{g}\left(S_{1}^{D}\right) \rightarrow \widetilde{E}_{D}$ is a holomorphic covering map. Since $\mathbb{H}$ is simplyconnected, any section $s$ of $\tau_{1}$ lifts to a section $\mathrm{Jac}^{-1} \circ s$ of $\tau_{11}$. Thus Theorem 5.1 immediately implies Theorem 6.1.

\section{Holomorphic motions}

In this section we use the theory of holomorphic motions to define and characterize the foliation $\mathcal{F}_{D}$.

Holomorphic motions. Given a set $E \subset \widehat{\mathbb{C}}$ and a basepoint $s \in \mathbb{H}$, a holomorphic motion of $E$ over $(\mathbb{H}, s)$ is a family of injective maps

$$
F_{t}: E \rightarrow \widehat{\mathbb{C}}, \quad t \in \mathbb{H},
$$

such that $F_{s}(z)=z$ and $F_{t}(z)$ is a holomorphic function of $t$.

A holomorphic motion of $E$ has a unique extension to a holomorphic motion of its closure $\bar{E}$; and each map $F_{t}: E \rightarrow \widehat{\mathbb{C}}$ extends to a quasiconformal homeomorphism of the sphere. In particular, $F_{t} \mid \operatorname{int}(E)$ is quasiconformal (see e.g. [Dou]).

These properties imply:

Theorem 7.1 Let $P$ be a partition of $\mathbb{H} \times \mathbb{H}$ into disjoint graphs of holomorphic functions. Then:

1. $P$ is the set of leaves of a transversally quasiconformal foliation $\mathcal{F}$ of $\mathbb{H} \times \mathbb{H}$; and

2. If we adjoin the graphs of the constant functions $f: \mathbb{H} \rightarrow \partial \mathbb{H}$ to $P$, we obtain a continuous foliation of $\mathbb{H} \times \overline{\mathbb{H}}$.

The foliation $\mathcal{F}_{\boldsymbol{D}}$. Recall that every component of $\widetilde{X}_{D}(1) \subset \mathbb{H} \times \mathbb{H}$ is the graph of a Möbius transformation. By Theorem 6.1, there is a unique partition of $\mathbb{H} \times \mathbb{H}-\widetilde{X}_{D}(1)$ into the graphs of holomorphic maps as well. 
Taken together, these graphs form the leaves of a foliation $\widetilde{\mathcal{F}}_{D}$ of $\mathbb{H} \times \mathbb{H}$ by the preceding result. Since $\widetilde{X}_{D}(1)$ is invariant under $\operatorname{SL}\left(\mathcal{O}_{D} \oplus \mathcal{O}_{D}^{\vee}\right)$, the foliation $\widetilde{\mathcal{F}}_{D}$ descends to a foliation $\mathcal{F}_{D}$ of $X_{D}$.

To characterize $\mathcal{F}_{D}$, recall that the surface $X_{D}$ admits a holomorphic involution $\iota\left(\tau_{1}, \tau_{2}\right)=\left(\tau_{2}, \tau_{1}\right)$ which preserves $X_{D}(1)$.

Theorem 7.2 The only leaves shared by $\mathcal{F}_{D}$ and $\iota\left(\mathcal{F}_{D}\right)$ are the curves in $X_{D}(1)$.

Proof. Let $f: \mathbb{H} \rightarrow \mathbb{H}$ be a holomorphic function whose graph $F$ is both a leaf of $\widetilde{\mathcal{F}}_{D}$ and $\iota\left(\widetilde{\mathcal{F}}_{D}\right)$. Then $\iota(F)$ is also a graph, so $f$ is an isometry. But if $F \cap \widetilde{X}_{D}(1)=\emptyset$, then $F$ lifts to a leaf of the foliation $\mathcal{F}_{1}$ of Teichmüller space, and hence $f$ is a contraction by [Mc4, Thm. 4.2].

Corollary 7.3 The only leaves of $\widetilde{\mathcal{F}}_{D}$ that are graphs of Möbius transformations are those belonging to $\tilde{X}_{D}(1)$.

Complex geodesics. Let us say $\mathcal{F}$ is a foliation by complex geodesics if each leaf is a hyperbolic Riemann surface, isometrically immersed for the Kobayashi metric. We can then characterize $\mathcal{F}_{D}$ as follows.

Theorem 7.4 Up to the action of $\iota, \mathcal{F}_{D}$ is the unique extension of the lamination $X_{D}(1)$ to a foliation of $X_{D}$ by complex geodesics.

Proof. Let $\mathcal{F}$ be a foliation by complex geodesics extending $X_{D}(1)$. Then every leaf of its lift $\widetilde{\mathcal{F}}$ to $\widetilde{X}_{D}$ is a Kobayashi geodesic for $\mathbb{H} \times \mathbb{H}$. But a complex geodesic in $\mathbb{H} \times \mathbb{H}$ is either the graph of a holomorphic function or its inverse, so every leaf belongs to either $\widetilde{\mathcal{F}}_{D}$ or $\iota\left(\widetilde{\mathcal{F}}_{D}\right)$. Consequently every leaf of $\mathcal{F}$ is a leaf of $\mathcal{F}_{D}$ or $\iota\left(\mathcal{F}_{D}\right)$. Since these foliations have no leaves in common on the open set $U=X_{D}-X_{D}(1), \mathcal{F}$ coincides with one or the other.

Stable curves. The Abelian varieties $E \times F$ in $X_{D}(1)$ are the Jacobians of certain stable curves with real multiplication, namely the nodal curves $Y=E \vee F$ obtained by gluing $E$ to $F$ at a single point. If we adjoin these stable curves to $\mathcal{M}_{2}$, we obtain a partial compactification $\mathcal{M}_{2}^{*}$ which maps isomorphically to $\mathcal{A}_{2}$. The locus $X_{D}(1)$ can then be regarded as the projection to $X_{D}$ of a finite set of $\mathrm{GL}_{2}^{+}(\mathbb{R})$ orbits in $\Omega \mathcal{M}_{2}^{*}$, giving another proof that it is a lamination. 


\section{Quasiconformal dynamics}

In this section we use the relative period map $\rho=\int_{y_{1}}^{y_{2}} \eta_{1}$ to define a meromorphic quadratic differential $q=(d \rho)^{2}$ transverse to $\mathcal{F}_{D}$. We then show the transverse dynamics of $\mathcal{F}_{D}$ is given by Teichmüller mappings relative to $q$.

Absolute periods. The level sets of $\tau_{1}$ form the leaves of a holomorphic foliation $\widetilde{\mathcal{A}}_{D}$ on $\mathbb{H} \times \mathbb{H}$ which covers foliation $\mathcal{A}_{D}$ of $X_{D}$. By (3.2), every $\tau=\left(\tau_{1}, \tau_{2}\right)$ determines a pair of eigenforms $\eta_{1}, \eta_{2} \in \Omega\left(A_{\tau}\right)$ such that the absolute periods

$$
\int_{C} \eta_{1}, \quad C \in H_{1}\left(A_{\tau}, \mathbb{Z}\right)
$$

are constant along the leaves of $\widetilde{\mathcal{A}}_{D}$. Since every leaf of $\widetilde{\mathcal{F}}_{D}$ is the graph of a function $f: \mathbb{H} \rightarrow \mathbb{H}$, we have:

Theorem 8.1 The foliation $\mathcal{A}_{D}$ is transverse to $\mathcal{F}_{D}$.

The Weierstrass curve. Recall that $E_{D} \subset X_{D}$ denotes the locus of Jacobians with real multiplication by $\mathcal{O}_{D}$. For $\left[A_{\tau}\right]=\operatorname{Jac}(Y) \in E_{D}$ we can regard the eigenforms $\eta_{1}, \eta_{2}$ as holomorphic 1-forms in $\Omega(Y) \cong \Omega\left(A_{\tau}\right)$.

Let $W_{D} \subset E_{D}$ denote the locus where $\eta_{1}$ has a double zero on $Y$. By [Mc5] we have:

Theorem 8.2 The locus $W_{D}$ is an algebraic curve with one or two irreducible components, each of which is a leaf of $\mathcal{F}_{D}$.

We refer to $W_{D}$ as the Weierstrass curve, since $\eta_{1}$ vanishes at a Weierstrass point of $Y$.

Relative periods. Let $E_{D}(1,1)=X_{D}-\left(W_{D} \cup X_{D}(1)\right)$ denote the Zariski open set where $\eta_{1}$ has a pair of simple zeros, and let $\widetilde{E}_{D}(1,1)$ be its preimage in the universal cover $\widetilde{X}_{D}$. Let

$$
\mathbb{H}_{s}=\{s\} \times \mathbb{H} \subset \mathbb{H} \times \mathbb{H},
$$

and let $\mathbb{H}_{s}^{*}=\mathbb{H}_{s} \cap \widetilde{E}_{D}(1,1)$.

For each $\tau \in \mathbb{H}_{s}^{*}$, let $y_{1}, y_{2}$ denote the zeros of the associated form $\eta_{1} \in$ $\Omega(Y)$. We can then define the (multivalued) relative period map $\rho_{s}: \mathbb{H}_{s}^{*} \rightarrow \mathbb{C}$ by

$$
\rho_{s}(\tau)=\int_{y_{1}}^{y_{2}} \eta_{1} .
$$


To make $\rho_{s}(\tau)$ single-valued, we must (locally) choose (i) an ordering of the zeros $y_{1}$ and $y_{2}$, and (ii) a path on $Y$ connecting them.

Quadratic differentials. Let $z$ be a local coordinate on $\mathbb{H}_{s}$, and recall that the absolute periods of $\eta_{1}$ are constant along $\mathbb{H}_{s}$. Thus if we change the choice of path from $y_{1}$ to $y_{2}$, the derivative $d \rho / d z$ remains the same; and if we interchange $y_{1}$ and $y_{2}$, it changes only by sign. Thus the quadratic differential

$$
q=(d \rho / d z)^{2} d z^{2}
$$

is globally well-defined on $\mathbb{H}_{s}^{*}$.

Theorem 8.3 The form $q$ extends to a meromorphic quadratic differential on $\mathbb{H}_{s}$, with simple zeros where $\mathbb{H}_{s}$ meets $\widetilde{W}_{D}$, and simple poles where it meets $\tilde{X}_{D}(1)$.

Proof. It is a general result that the period map provides holomorphic local coordinates on any stratum of $\Omega \mathcal{M}_{g}$ (see [V2], [MS, Lemma 1.1], [KZ]). Thus $\rho_{s} \mid \mathbb{H}_{s}^{*}$ is holomorphic with $d \rho_{s} \neq 0$, and hence $q \mid \mathbb{H}_{s}^{*}$ is a nowhere vanishing holomorphic quadratic differential.

To see $q$ acquires a simple zero when $\eta_{1}$ acquires a double zero, note that the relative period map

$$
\rho(t)=\int_{-\sqrt{t}}^{\sqrt{t}}\left(z^{2}-t\right) d z=(-4 / 3) t^{3 / 2}
$$

of the local model $\eta_{t}=\left(z^{2}-t\right) d z$ satisfies $(d \rho / d t)^{2}=4 t$. Similarly, a point of $\mathbb{H}_{s} \cap \widetilde{X}_{D}(1)$ is locally modeled by the family of connected sums

$$
\left(Y_{t}, \eta_{t}\right)=\left(E_{1}, \omega_{1}\right) \underset{I}{\#}\left(E_{2}, \omega_{2}\right)
$$

with $I=[0, \rho(t)]=[0, \pm \sqrt{t}]$. Since $(d \rho / d t)^{2}=1 /(4 t)$, at these points $q$ has simple poles.

See $[\mathrm{Mc} 7, \S 6]$ for more on connected sums.

Teichmüller maps. Now let $f: \mathbb{H}_{s} \rightarrow \mathbb{H}_{t}$ be a quasiconformal map. We say $f$ is a Teichmüller map, relative to a holomorphic quadratic differential $q$, if its complex dilatation satisfies

$$
\mu(f)=\left(\frac{\partial f / \partial \bar{z}}{\partial f / \partial z}\right) \frac{d \bar{z}}{d z}=\alpha \frac{\bar{q}}{|q|}
$$


for some $\alpha \in \mathbb{C}^{*}$. This is equivalent to the condition that $w=f(z)$ is real-linear in local coordinates where $q=d z^{2}$ and $d w^{2}$ respectively. In such charts we can write

$$
w=w_{0}+D_{q}(f) \cdot z,
$$

with $D_{q}(f) \in \mathrm{SL}_{2}(\mathbb{R})$. We refer to $D_{q}(f)$ as the linear part of $f$; it is only well-defined up to sign, since $z \mapsto-z$ preserves $d z^{2}$.

Theorem 8.4 Given $g=\left(\begin{array}{cc}a & b \\ c & d\end{array}\right) \in \operatorname{SL}\left(\mathcal{O}_{D} \oplus \mathcal{O}_{D}^{\vee}\right)$ and $s \in \mathbb{H}$, let $\mathbb{H}_{t}=g\left(\mathbb{H}_{s}\right)$. Then the linear part of $g: \mathbb{H}_{s} \rightarrow \mathbb{H}_{t}$ is given by $D_{q}(g) \cdot z=(d-c s)^{-1} z$.

Proof. Since the Riemann surfaces $Y$ at corresponding points of $\mathbb{H}_{s}$ and $\mathbb{H}_{t}$ differ only by marking, the relative period maps $\rho_{s}$ and $\rho_{t}$ differ only by the normalization of $\eta_{1}$. This discrepancy is accounted for by equation (3.4), which gives $\rho_{t} / \rho_{s}=\chi(g, s)=(d-c s)^{-1}$. Since the coordinates $\rho_{s}$ and $\rho_{t}$ linearize $q$, the map $D_{q}(g)$ is given by multiplication by $(d-c s)^{-1}$.

Now let $C_{s t}: \mathbb{H}_{s} \rightarrow \mathbb{H}_{t}$ be the unique map such that $z$ and $C_{s t}(z)$ lie on the same leaf of $\widetilde{\mathcal{F}}_{D}$.

Theorem 8.5 The linear part of $C_{s t}$ is given by $D_{q}\left(C_{s t}\right)=A_{t} A_{s}^{-1}$, where $A_{u}=\left(\begin{array}{cc}1 & \operatorname{Re}(u) \\ 0 & \operatorname{Im}(u)\end{array}\right) \in \operatorname{PSL}_{2}(\mathbb{R})$.

Proof. By the definition of $\mathcal{F}_{D}$, the forms $\eta_{1}$ at corresponding points of $\mathbb{H}_{s}^{*}$ and $\mathbb{H}_{t}^{*}$ are related by some element $B \in \mathrm{GL}_{2}^{+}(\mathbb{R})$ acting on $\Omega \mathcal{T}_{g}$. Thus $\rho_{t}=B \circ \rho_{s}$ and therefore $D_{q}\left(C_{s t}\right)=B$. Since the action of $B$ on the absolute periods of $\eta_{1}$ satisfies

$$
B\left(\mathcal{O}_{D} \oplus \mathcal{O}_{D}^{\vee} s\right)=\mathcal{O}_{D} \oplus \mathcal{O}_{D}^{\vee} t
$$

(in the sense of equation (3.1)), we have $B(1)=1$ and $B(s)=t$, and thus $B=A_{t} A_{s}^{-1}$ as above.

Dynamics. Every leaf of $\widetilde{\mathcal{F}}_{D}$ meets the transversal $\mathbb{H}_{s}$ in a single point. Thus the action of $g \in \operatorname{SL}\left(\mathcal{O}_{D} \oplus \mathcal{O}_{D}^{\vee}\right)$ on the space of leaves determines a holonomy map

$$
\phi_{g}: \mathbb{H}_{s} \rightarrow \mathbb{H}_{s},
$$

characterized by the property that $\left(s, \phi_{g}(z)\right)$ lies on the same leaf as $g(s, z)$.

Theorem 8.6 The group $\operatorname{SL}\left(\mathcal{O}_{D} \oplus \mathcal{O}_{D}^{\vee}\right)$ acts on $\mathbb{H}_{s}$ by Teichmüller mappings, satisfying $D_{q}\left(\phi_{g}\right)=g$ in the case $s=i$. 
(As usual we regard $g$ as a real matrix using $\iota_{1}: K \rightarrow \mathbb{R}$.)

Proof. Let $g=\left(\begin{array}{ll}a & b \\ c & d\end{array}\right)$, and $t=(a s-b) /(-c s+d)$; then $\mathbb{H}_{t}=g\left(\mathbb{H}_{s}\right)$.

Since $\phi_{g}(z)$ is obtained from $g(s, z)$ by combing it along the leaves of $\widetilde{\mathcal{F}}_{D}$ back into $\mathbb{H}_{s}$, we have $\phi_{g}(s, z)=C_{t s}(g(s, z))$. Thus the chain rule implies

$$
D_{q}\left(\phi_{g}\right) \cdot z=B \cdot z=A_{s} \circ A_{t}^{-1}(z /(-c s+d)) .
$$

Now assume $s=i$. Then we have $B(a i-b)=A_{t}^{-1}(t)=i$ and $B(-c i+d)=$ $A_{t}^{-1}(1)=1$; therefore $B^{-1}=\left(\begin{array}{cc}d & -b \\ -c & a\end{array}\right)$ and thus $B=\left(\begin{array}{ll}a & b \\ c & d\end{array}\right)=g$.

Corollary 8.7 The foliation $\mathcal{F}_{D}$ carries a natural transverse invariant measure.

Proof. Since $\operatorname{det} D_{q}\left(\phi_{g}\right)=1$ for all $g$, the form $|q|$ gives a holonomyinvariant measure on the transversal $\mathbb{H}_{s}$.

Finally we show that, although $\phi_{g} \mid \mathbb{H}_{s}$ is quasiconformal, its continuous extension to $\partial \mathbb{H}_{s}$ is a Möbius transformation.

Theorem 8.8 For any $g=\left(\begin{array}{ll}a & b \\ c & d\end{array}\right) \in \operatorname{SL}\left(\mathcal{O}_{D} \oplus \mathcal{O}_{D}^{\vee}\right)$ and $z \in \partial \mathbb{H}_{s}$, we have

$$
\phi_{g}(z)=\left(a^{\prime} z-b^{\prime}\right) /\left(-c^{\prime} z+d^{\prime}\right) .
$$

Proof. By Theorem 7.1, the combing maps $C_{s t}$ extend to the identity on $\partial \mathbb{H}_{s}$. Thus $\left(t, \phi_{g}(z)\right)=g(s, z)$, and the result follows from equation (3.3).

Note: if we use the transversal $\mathbb{H}_{t}$ instead of $\mathbb{H}_{s}$, the holonomy simply changes by conjugation by $C_{s t}$.

\section{$9 \quad$ Further results}

In this section we summarize related results on the density of leaves, isoperiodic forms, holomorphic motions and iterated rational maps.

I. Density of leaves. By [Mc7], the closure of the complex geodesic $f$ : $\mathbb{H} \rightarrow \mathcal{M}_{2}$ generated by a holomorphic 1 -form is either an algebraic curve, a Hilbert modular surface or the whole moduli space. Since the leaves of $\mathcal{F}_{D}$ are examples of such complex geodesics, we obtain: 
Theorem 9.1 Every leaf of $\mathcal{F}_{D}$ is either a closed algebraic curve, or a dense subset of $X_{D}$.

It is easy to see that the union of the closed leaves is dense when $D=d^{2}$. On the other hand, the classification of Teichmüller curves in [Mc5] and [Mc6] implies:

Theorem 9.2 If $D$ is not a square, then $\mathcal{F}_{D}$ has only finitely many closed leaves. These consist of the components of $W_{D} \cup X_{D}(1)$ and, when $D=5$, the Teichmüller curve generated by the regular decagon.

II. Isoperiodic forms. Next we discuss interactions between the foliations $\mathcal{F}_{D}$ and $\mathcal{A}_{D}$. When $D=d^{2}$ is a square, the surface $X_{D}$ is finitely covered by a product, and hence every leaf of $\mathcal{A}_{D}$ is closed.

Theorem 9.3 If $D$ is not a square, then every leaf $L$ of $\mathcal{A}_{D}$ is dense in $X_{D}$, and $L \cap F$ is dense in $F$ for every leaf $F$ of $\mathcal{F}_{D}$.

Proof. The first result follows from the fact that $\operatorname{SL}\left(\mathcal{O}_{D} \oplus \mathcal{O}_{D}^{\vee}\right)$ is a dense subgroup of $\mathrm{SL}_{2}(\mathbb{R})$, and the second follows from the first by transversality of $\mathcal{A}_{D}$ and $\mathcal{F}_{D}$.

Let us say a pair of 1 -forms $\left(Y_{i}, \omega_{i}\right) \in \Omega \mathcal{M}_{g}$ are isoperiodic if there is a symplectic isomorphism

$$
\phi: H_{1}\left(Y_{1}, \mathbb{Z}\right) \rightarrow H_{1}\left(Y_{2}, \mathbb{Z}\right)
$$

such that the period maps

$$
I\left(\omega_{i}\right): H_{1}\left(Y_{i}, \mathbb{Z}\right) \rightarrow \mathbb{C}
$$

satisfy $I\left(\omega_{1}\right)=I\left(\omega_{2}\right) \circ \phi$. Since the absolute periods of $\eta_{1}$ are constant along the leaves of $\mathcal{A}_{D}$, from the preceding result we obtain:

Corollary 9.4 The $\mathrm{SL}_{2}(\mathbb{R})$-orbit of any eigenform for real multiplication by $\mathcal{O}_{D}, D \neq d^{2}$, contains infinitely many isoperiodic forms.

For a concrete example, let $Q \subset \mathbb{C}$ be a regular octagon containing $[0,1]$ as an edge. Identifying opposite sides of $Q$, we obtain the octagonal form

$$
(Y, \omega)=(Q, d z) / \sim
$$


of genus two.

Let $\mathbb{Z}[\zeta] \subset \mathbb{C}$ denote the ring generated by $\zeta=(1+i) / \sqrt{2}=\exp (2 \pi i / 8)$, equipped with the symplectic form

$$
\left\langle z_{1}, z_{2}\right\rangle=\operatorname{Tr}_{\mathbb{Q}}^{\mathbb{Q}(\zeta)}\left(\left(\zeta+\zeta^{2}+\zeta^{3}\right) z_{1} \bar{z}_{2} / 4\right)
$$

Then it is easy to check that:

1. The octagonal form $\omega$ has a single zero of order 2 , and

2. Its period map $I(\omega)$ sends $H_{1}(Y, \mathbb{Z})$ to $\mathbb{Z}[\zeta]$ by a symplectic isomorphism.

However, these two properties do not determine $(Y, \omega)$ uniquely. Indeed, $\omega$ is an eigenform for real multiplication by $\mathcal{O}_{8}$, so the preceding Corollary ensures there are infinitely many isoperiodic forms $\left(Y_{i}, \omega_{i}\right)$ in its $\mathrm{SL}_{2}(\mathbb{R})$ orbit. In other words we have:

Corollary 9.5 There are infinite many fake octagonal forms in $\Omega \mathcal{M}_{2}$.

Note that the forms $\left(Y_{i}, \omega_{i}\right)$ cannot be distinguished by their relative periods either, since they all have double zeros.

A similar statement can be formulated for the pentagonal form on the curve $y^{2}=x^{5}-1$.

III. Top-speed motions. Let $F_{t}: E \rightarrow \mathbb{H}$ be a holomorphic motion of $E \subset \mathbb{H}$ over $(\mathbb{H}, s)$. By the Schwarz lemma, we have $\left\|d F_{t}(z) / d t\right\| \leq 1$ with respect to the hyperbolic metric on $\mathbb{H}$. Let us say $F_{t}$ is a top-speed holomorphic motion if equality holds everywhere; equivalently, if $t \mapsto F_{t}(z)$ is an isometry of $\mathbb{H}$ for every $z \in E$.

A top-speed holomorphic motion is maximal if it cannot be extended to a top-speed motion of a larger set $E^{\prime} \supset E$.

Theorem 9.6 For any discriminant $D \geq 4$, the map

$$
F_{t}(U(s))=U(t), \quad U \in \Lambda_{D}
$$

gives a maximal top-speed holomorphic motion of $E=\Lambda_{D} \cdot s$ over $(\mathbb{H}, s)$.

Proof. Let $t \mapsto f(t)=F_{t}(z)$ be an extension of the motion to a point $z \notin E$. Then the graph of $f$ is a leaf of $\widetilde{\mathcal{F}}_{D}$, since it is disjoint from $\widetilde{X}_{D}(1)$. But the only leaves that are graphs of Möbius transformations are those in $\widetilde{X}_{D}(1)$, by Corollary 7.3 . 
Corollary 9.7 The group $\Gamma(2)=\left\{A \in \mathrm{SL}_{2}(\mathbb{Z}): A \equiv I \bmod 2\right\}$ gives a maximal top-speed holomorphic motion of $E=\Gamma(2) \cdot s$ over $(\mathbb{H}, s)$.

Proof. We have $\Gamma(2)=g \Lambda_{4} g^{-1}$, where $g=\left(\begin{array}{cc}1 / 2 & 0 \\ 0 & 2\end{array}\right)$ (Theorem 4.4).

IV. Iterated rational maps. Finally we explain how the foliation $\mathcal{F}_{4}$ of $X_{4}$ arises in complex dynamics.

First recall that the moduli space of elliptic curves can be described as the quotient orbifold $\mathcal{M}_{1}=\widetilde{\mathcal{M}}_{1} / S_{3}$, where

$$
\widetilde{\mathcal{M}}_{1}=\mathbb{H} / \Gamma(2) \cong \mathbb{C}-\{0,1\} .
$$

The deck group $S_{3}$ also acts diagonally on $\widetilde{\mathcal{M}}_{1} \times \widetilde{\mathcal{M}}_{1}$, preserving the diagonal $\Delta$.

Theorem 9.8 For $D=4$, we have $\left(X_{D}, X_{D}(1)\right) \cong\left(\widetilde{\mathcal{M}}_{1} \times \widetilde{\mathcal{M}}_{1}, \Delta\right) / S_{3}$.

Proof. Since $\mathcal{O}_{4}^{\vee}=(1 / 2) \mathcal{O}_{4}$, the surface $X_{4}$ is isomorphic to $(\mathbb{H} \times \mathbb{H}) / \mathrm{SL}_{2}\left(\mathcal{O}_{4}\right)$. In these coordinates we have $\Lambda_{4}=\Gamma(2)$. Since

$$
\mathrm{SL}_{2}\left(\mathcal{O}_{4}\right) \cong\left\{\left(A_{1}, A_{2}\right) \in \mathrm{SL}_{2}(\mathbb{Z}): A_{1} \equiv A_{2} \bmod 2\right\}
$$

contains $\Gamma(2) \times \Gamma(2)$ as a subgroup of index 6 , the result follows.

Now consider, for each $t \in \widetilde{\mathcal{M}}_{1}$, the elliptic curve $E_{t}$ defined by $y^{2}=$ $x(x-1)(x-t)$. There is a unique rational map $f_{t}: \mathbb{P}^{1} \rightarrow \mathbb{P}^{1}$ such that

$$
x(2 P)=f_{t}(x(P))
$$

with respect to the usual group law on $E_{t}$. Indeed, using the fact that $-2 P$ lies on the tangent line to $E_{t}$ at $P$, we find

$$
f_{t}(z)=\frac{\left(z^{2}-t\right)^{2}}{4 z(z-1)(z-t)} .
$$

Note that the postcritical set

$$
P\left(f_{t}\right)=\bigcup\left\{f_{t}^{n}(z): n>0, f_{t}^{\prime}(z)=0\right\}
$$

coincides with the branch locus $\{0,1, t, \infty\}$ of the map $x: E_{t} \rightarrow \mathbb{P}^{1}$.

The rational maps $f_{t}(z)$ form a stable family of Lattès examples. It is well-known that the Julia set of any Lattès example is the whole Riemann sphere; and that in any stable family, the Julia set varies by a holomorphic motion respecting the dynamics (see e.g. [MSS], [Mc1, Ch. 4], [Mil].) 
Theorem 9.9 As $t$ varies in $\widetilde{\mathcal{M}}_{1}$, the holomorphic motion of $J\left(f_{t}\right)$ sweeps out the lift of the foliation $\mathcal{F}_{4}$ to the covering space $\widetilde{\mathcal{M}}_{1} \times \widetilde{\mathcal{M}}_{1}$ of $X_{4}$.

Proof. Let $\mathcal{G}$ be the foliation of $\widetilde{\mathcal{M}}_{1} \times \mathbb{P}^{1}$ swept out by $J\left(f_{t}\right)$. Since the holomorphic motion respects the dynamics, it preserves the post-critical set, and thus the leaves of $\mathcal{G}$ include the loci $z=0,1, \infty$ as well as the diagonal $t=z$. In particular, $\mathcal{G}$ restricts to a foliation of the finite cover $\widetilde{\mathcal{M}}_{1} \times \widetilde{\mathcal{M}}_{1}-\Delta$ of $X_{4}-X_{4}(1)$. Since each leaf of $\mathcal{G}$ lifts to the graph of a holomorphic function in the universal cover $\mathbb{H} \times \mathbb{H}$, it lies over a leaf of $\mathcal{F}_{D}$ by the uniqueness part of Theorem 1.2.

Algebraic curves. The loci $f_{t}^{n}(z)=\infty$ form a dense set of algebraic leaves of $\mathcal{G}$ that can easily be computed inductively. The real points of these curves are graphed in Figure 1; thus the figure depicts the lift of $\mathcal{F}_{4}$ to the finite cover $\widetilde{\mathcal{M}}_{1} \times \widetilde{\mathcal{M}}_{1}$ of $X_{4}$.

\section{References}

[Ah] L. Ahlfors. The complex analytic structure of the space of closed Riemann surfaces. In Analytic Functions, pages 45-66. Princeton Univ. Press, 1960.

[BR] L. Bers and H. L. Royden. Holomorphic families of injections. Acta Math. 157(1986), 259-286.

[BL] C. Birkenhake and H. Lange. Complex Abelian Varieties. SpringerVerlag, 1992.

[Br1] M. Brunella. Feuilletages holomorphes sur les surfaces complexes compactes. Ann. Sci. École Norm. Sup. 30(1997), 569-594.

[Br2] M. Brunella. Subharmonic variation of the leafwise Poincaré metric. Invent. math. 152(2003), 119-148.

[Dou] A. Douady. Prolongement de mouvements holomorphes (d'après Słodkowski et autres). In Séminaire Bourbaki, 1993/94, pages 720. Astérisque, vol. 227, 1995.

[EKK] C. J. Earle, I. Kra, and S. L. Krushkal. Holomorphic motions and Teichmüller spaces. Trans. Amer. Math. Soc. 343(1994), 927-948. 
[Gd] F. Gardiner. Teichmüller Theory and Quadratic Differentials. Wiley Interscience, 1987.

[vG] G. van der Geer. Hilbert Modular Surfaces. Springer-Verlag, 1987.

[GKZ] B. H. Gross, W. Kohnen, and D. Zagier. Heegner points and derivatives of $L$-series. Math. Ann. 278(1987), 497-562.

[HH] W. F. Hammond and F. Hirzebruch. L-series, modular embeddings and signatures. Math. Ann. 204(1973), 263-270.

[Hir] F. Hirzebruch. Hilbert modular surfaces. Enseign. Math. 19(1973), $183-281$.

[HZ1] F. Hirzebruch and D. Zagier. Intersection numbers of curves on Hilbert modular surfaces and modular forms of Nebentypus. Invent. math. 36(1976), 57-113.

[HZ2] F. Hirzebruch and D. Zagier. Classification of Hilbert modular surfaces. In Complex Analysis and Algebraic Geometry, pages 43-77. Cambridge University Press and Iwanami Shoten, 1977.

[KZ] M. Kontsevich and A. Zorich. Connected components of the moduli spaces of Abelian differentials with prescribed singularities. Invent. math. 153(2003), 631-678.

[MR] C. Maclachlan and A. W. Reid. The Arithmetic of Hyperbolic 3Manifolds. Springer-Verlag, 2003.

[MSS] R. Mañé, P. Sad, and D. Sullivan. On the dynamics of rational maps. Ann. Sci. Éc. Norm. Sup. 16(1983), 193-217.

[Mn] W. R. Mann. Gross-Zagier revisited (Appendix). In Heegner Points and Rankin L-Series, volume 49 of Math. Sci. Res. Inst. Publ., pages 139-162. Cambridge Univ. Press, 2004.

[MS] H. Masur and J. Smillie. Hausdorff dimension of sets of nonergodic measured foliations. Annals of Math. 134(1991), 455-543.

[Mc1] C. McMullen. Complex Dynamics and Renormalization, volume 135 of Annals of Math. Studies. Princeton University Press, 1994.

[Mc2] C. McMullen. The classification of conformal dynamical systems. In Current Developments in Mathematics, 1995, pages 323-360. International Press, 1995. 
[Mc3] C. McMullen. Polynomial invariants for fibered 3-manifolds and Teichmüller geodesics for foliations. Ann. scient. Éc. Norm. Sup. 33(2000), 519-560.

[Mc4] C. McMullen. Billiards and Teichmüller curves on Hilbert modular surfaces. J. Amer. Math. Soc. 16(2003), 857-885.

[Mc5] C. McMullen. Teichmüller curves in genus two: Discriminant and spin. Math. Ann. 333(2005), 87-130.

[Mc6] C. McMullen. Teichmüller curves in genus two: Torsion divisors and ratios of sines. Invent. math. 165(2006), 651-672.

[Mc7] C. McMullen. Dynamics of $\mathrm{SL}_{2}(\mathbf{R})$ over moduli space in genus two. Annals of Math., To appear.

[McS] C. McMullen and D. Sullivan. Quasiconformal homeomorphisms and dynamics III: The Teichmüller space of a holomorphic dynamical system. Adv. Math. 135(1998), 351-395.

[Mil] J. Milnor. On Lattès maps. In P. G. Hjorth and C. L. Petersen, editors, Dynamics on the Riemann sphere, pages 9-44. European Math. Soc., 2006.

[Ru] B. Runge. Endomorphism rings of abelian surfaces and projective models of their moduli spaces. Tôhoku Math. J. 51(1999), 283-303.

[Sl] Z. Słodkowski. Holomorphic motions and polynomial hulls. Proc. Amer. Math. Soc. 111(1991), 347-355.

[Sul] D. Sullivan. Quasiconformal homeomorphisms and dynamics II: Structural stability implies hyperbolicity for Kleinian groups. Acta Math. 155(1985), 243-260.

[V1] W. Veech. Teichmüller curves in moduli space, Eisenstein series and an application to triangular billiards. Invent. math. 97(1989), 553583.

[V2] W. Veech. Moduli spaces of quadratic differentials. J. Analyse Math. 55(1990), 117-171.

[Vi] M.-F. Vigneras. Arithmétique des algèbres de quaternions, volume 800 of Lectures Notes in Mathematics. Springer-Verlag, 1980. 
Mathematics Department

HARVARD UNIVERSITY

1 OXFord ST

CAmbridge, MA 02138-2901 